Research Article

\title{
Development and Validation of a Robust Immune-Related Prognostic Signature for Gastric Cancer
}

\author{
Junyu Huo $\mathbb{D}^{1,2}$ Liqun $W u \mathbb{D}^{1},{ }^{1}$ and Yunjin Zang $\mathbb{D}^{1}$ \\ ${ }^{1}$ Liver Disease Center, The Affiliated Hospital of Qingdao University, No. 59 Haier Road, Qingdao 266003, China \\ ${ }^{2}$ Qingdao University, No. 308 Ningxia Road, Qingdao 266071, China
}

Correspondence should be addressed to Liqun Wu; wulq5810@126.com

Received 31 January 2021; Revised 11 April 2021; Accepted 13 April 2021; Published 3 May 2021

Academic Editor: Xiaofeng Yang

Copyright (C) 2021 Junyu Huo et al. This is an open access article distributed under the Creative Commons Attribution License, which permits unrestricted use, distribution, and reproduction in any medium, provided the original work is properly cited.

\begin{abstract}
Background. An increasing number of reports have found that immune-related genes (IRGs) have a significant impact on the prognosis of a variety of cancers, but the prognostic value of IRGs in gastric cancer (GC) has not been fully elucidated. Methods. Univariate Cox regression analysis was adopted for the identification of prognostic IRGs in three independent cohorts (GSE62254, $n=300$; GSE15459, $n=191$; and GSE26901, $n=109$ ). After obtaining the intersecting prognostic genes, the three independent cohorts were merged into a training cohort $(n=600)$ to establish a prognostic model. The risk score was determined using multivariate Cox and LASSO regression analyses. Patients were classified into low-risk and high-risk groups according to the median risk score. The risk score performance was validated externally in the three independent cohorts (GSE26253, $n=432$; GSE84437, $n=431$; and TCGA, $n=336$ ). Immune cell infiltration (ICI) was quantified by the CIBERSORT method. Results. A risk score comprising nine genes showed high accuracy for the prediction of the overall survival (OS) of patients with GC in the training cohort (AUC > 0.7). The risk of death was found to have a positive correlation with the risk score. The univariate and multivariate Cox regression analyses revealed that the risk score was an independent indicator of the prognosis of patients with GC $(p<0.001)$. External validation confirmed the universal applicability of the risk score. The low-risk group presented a lower infiltration level of M2 macrophages than the high-risk group $(p<0.001)$, and the prognosis of patients with GC with a higher infiltration level of M2 macrophages was poor $(p=0.011)$. According to clinical correlation analysis, compared with patients with the diffuse and mixed type of GC, those with the Lauren classification intestinal GC type had a significantly lower risk score $(p=0.00085)$. The patients' risk score increased with the progression of the clinicopathological stage. Conclusion. In this study, we constructed and validated a robust prognostic signature for GC, which may help improve the prognostic assessment system and treatment strategy for GC.
\end{abstract}

\section{Background}

Gastric cancer (GC) originates from the most superficial mucosal epithelial cells of the gastric wall [1]. GC development is a progressive process involving many factors and steps, such as genetic factors, Helicobacter pylori infections, dietary or environmental factors, and precancerous states $[2,3]$. The latest statistical results showed that GC ranked first among the malignant tumors of the digestive system, representing a serious threat to human health $[4,5]$. Although the overall survival (OS) of GC has been significantly increased in recent years due to the diversification of treatments, such as targeted therapy, angiogenic therapy, and immunotherapy [6], there is still much room for progress in improving the prognosis of GC with a surgery-based comprehensive treatment strategy.

Genetics were found to play a crucial role in the molecular mechanism responsible for GC occurrence and growth [7]. At present, many genes related to GC have been found. Some of these genes exist in the cell genome in the form of oncogenes, encode proteins required for cell growth, and drive carcinogenesis when they are activated by point mutation, translocation, and amplification [8]. Some of the genes are tumor suppressors, which play a negative regulatory role in controlling cell growth, proliferation, and differentiation [9]. Finally, some of the genes affect the biological process 


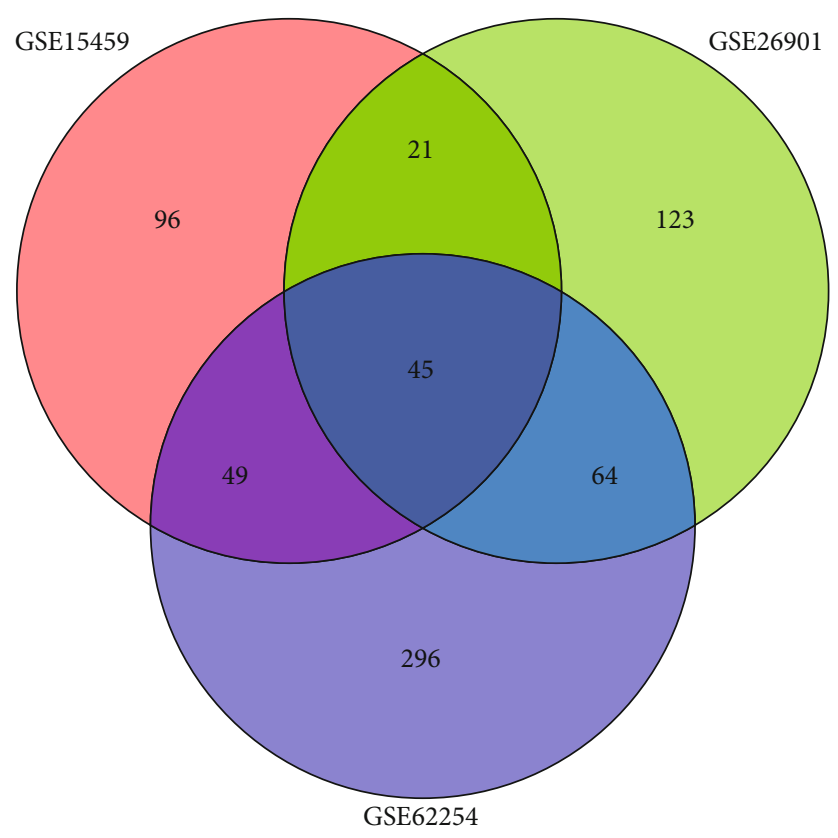

(a)

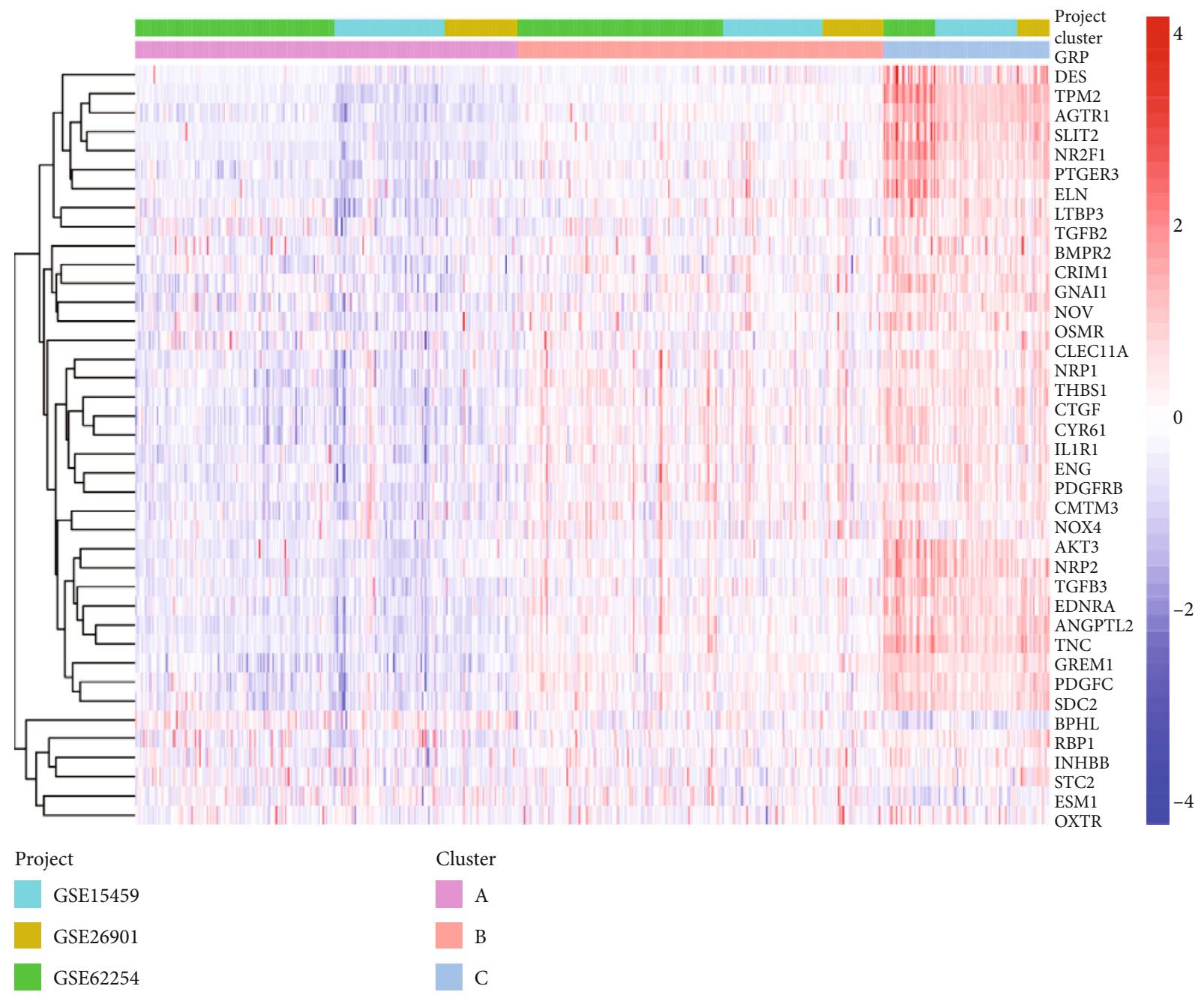

(b)

Figure 1: Continued. 


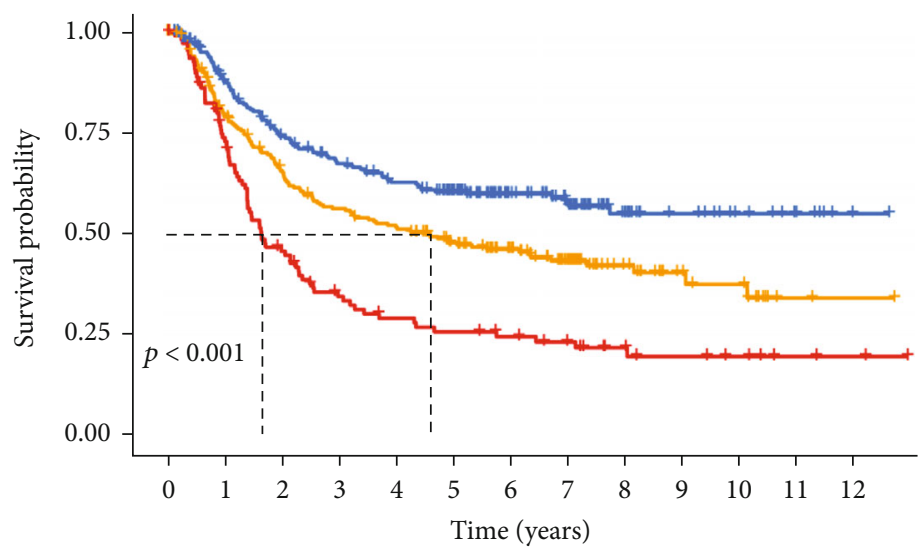

Number at risk

\begin{tabular}{|c|c|c|c|c|c|c|c|c|c|c|c|c|}
\hline 251 & 202 & 166 & 147 & 133 & 117 & 79 & 57 & 29 & 17 & 11 & 7 & 2 \\
\hline 240 & 180 & 143 & 122 & 112 & 90 & 71 & 50 & 26 & 15 & 12 & 2 & 1 \\
\hline 109 & 77 & 47 & 32 & 26 & 23 & 20 & 17 & 11 & 8 & 6 & 3 & 2 \\
\hline $\begin{array}{l}1 \\
0\end{array}$ & $\begin{array}{l}1 \\
1\end{array}$ & 2 & 3 & 4 & $\begin{array}{l}1 \\
5\end{array}$ & 6 & 7 & 8 & 9 & $\begin{array}{l}1 \\
10\end{array}$ & $\begin{array}{c}1 \\
11\end{array}$ & 12 \\
\hline
\end{tabular}

Gene cluster

$+\mathrm{A}$

$+\mathrm{B}$

$+\mathrm{C}$

(c)

Figure 1: Gene cluster analysis. (a) Venn plot of the 45 intersecting prognosis-related genes. (b) Heatmap of gene clusters. (c) Kaplan-Meier survival analysis regarding gene clusters and OS in the training cohort.

of GC by altering the drug resistance of the cancer cells [10]. Therefore, a full understanding of the function of genes in the progression of GC is expected to enrich our understanding of the therapeutic targets of GC.

A growing number of reports have revealed that immune-related genes (IRGs) have a significant impact on the prognosis of a variety of cancers [11-14]. However, the prognostic value of IRGs for GC has not been fully elucidated. In this study, we integrated and analyzed highthroughput sequencing data from public databases and provided a robust prognostic signature for GC based on nine IRGs. The predictive power of the prognostic signature was confirmed by internal and external validation. Meanwhile, we explored the prognostic mechanism of the signature by combining quantitative analysis regarding immune cell infiltration (ICI) with correlation analysis regarding clinical features.

\section{Materials and Methods}

2.1. Data Collection. The Cancer Genome Atlas (https:// portal.gdc.cancer.gov/) and the Gene Expression Omnibus (GEO) databases (https://www.ncbi.nlm.nih.gov/geo/) were used for the collection of gene expression profiles and clinical data. The data utilized in this study were obtained from public databases. We complied with the access rules of TCGA and GEO databases during the process of data collection, and it was unnecessary to obtain approval from the local ethics committee.
2.2. Identification of Prognostic Immune-Related Genes. We extracted data on the immune-related genes of three independent cohorts (GSE62254, $n=300$; GSE15459, $n=191$; and GSE26901, $n=109$ ) according to the immune-related gene list acquired from the ImmPort database (https:// immport.niaid.nih.gov). Next, we set $p<0.05$ as the screening criterion and screened the prognosis-related genes by univariate Cox regression analysis in the three independent cohorts.

2.3. Cluster Analysis of the Intersection Prognostic Genes. We merged GSEE62254, GSE15459, and GSE26901 into a training cohort $(n=600)$, and the batch effects between different datasets were removed by the "combat" function in the $\mathrm{R}$ package "sva." We conducted cluster analysis based on the $\mathrm{R}$ package "consensusclusterplus" to further analyze the prognostic value of overlapping prognostic genes.

2.4. Development and Validation of a Prognostic Signature. Based on the least absolute shrinkage and selection operator (LASSO) algorithm, we removed the overfitting between prognosis-related genes by 10 -fold cross-validation for penalty parameter tuning and kept genes that had nonzero regression coefficients for later multivariate Cox regression analyses $[15,16]$. Each gene's regression coefficient obtained from such analysis was multiplied by its expression level, thus resulting in the risk score $[16,17]$. Based on the median score, we divided patients with GC into low- and high-risk groups. The LASSO regression analysis was performed with 
TABLE 1: The 45 intersection prognosis-related genes identified by univariate Cox regression analysis in the training cohort.

\begin{tabular}{|c|c|c|c|c|}
\hline Gene list & Category & HR & HR.95L & HR.95H \\
\hline AGTR1 & Cytokine_receptors & 1.334317 & 1.217829 & 1.461947 \\
\hline AKT3 & BCRSignalingPathway & 1.761968 & 1.444244 & 2.149589 \\
\hline ANGPTL2 & Cytokine_receptors & 1.344887 & 1.192773 & 1.5164 \\
\hline BMPR2 & Cytokine_receptors & 2.362857 & 1.701484 & 3.28131 \\
\hline BPHL & Antimicrobials & 0.637487 & 0.504375 & 0.80573 \\
\hline CLEC11A & Cytokines & 1.516036 & 1.301901 & 1.765391 \\
\hline CMTM3 & Cytokines & 1.939969 & 1.499121 & 2.510458 \\
\hline CRIM1 & Cytokine_receptors & 1.923839 & 1.533551 & 2.413454 \\
\hline CTGF & Cytokines & 1.603191 & 1.381789 & 1.860068 \\
\hline CYR61 & Cytokines & 1.384955 & 1.224587 & 1.566325 \\
\hline DAK & Antimicrobials & 0.586742 & 0.442452 & 0.778088 \\
\hline DES & Antimicrobials & 1.205936 & 1.13654 & 1.279568 \\
\hline EDNRA & Chemokine_receptors & 1.499985 & 1.315628 & 1.710175 \\
\hline ELN & Antimicrobials & 1.490388 & 1.305397 & 1.701595 \\
\hline ENG & Cytokine_receptors & 1.391229 & 1.18348 & 1.635447 \\
\hline ESM1 & Cytokines & 1.462723 & 1.244433 & 1.719303 \\
\hline GNAI1 & Antimicrobials & 1.427925 & 1.196914 & 1.703522 \\
\hline GREM1 & Cytokines & 1.317087 & 1.181972 & 1.467647 \\
\hline GRP & Cytokines & 1.227876 & 1.140799 & 1.321601 \\
\hline HDGFRP3 & Cytokines & 1.603254 & 1.380716 & 1.86166 \\
\hline IL1R1 & Cytokine_receptors & 1.613095 & 1.373481 & 1.894511 \\
\hline INHBB & TGFb_family_member & 1.544374 & 1.362625 & 1.750365 \\
\hline LTBP3 & Cytokines & 1.722496 & 1.358221 & 2.184469 \\
\hline $\mathrm{NOV}$ & Cytokines & 1.563551 & 1.372153 & 1.781646 \\
\hline NOX4 & Antimicrobials & 1.639326 & 1.41234 & 1.902793 \\
\hline NPR3 & Cytokine_receptors & 1.849908 & 1.547669 & 2.21117 \\
\hline $\mathrm{NR} 2 \mathrm{~F} 1$ & Cytokine_receptors & 1.378966 & 1.224325 & 1.55314 \\
\hline NRP1 & Cytokine_receptors & 1.707208 & 1.420286 & 2.052093 \\
\hline NRP2 & Cytokine_receptors & 1.691583 & 1.393531 & 2.053385 \\
\hline OSMR & Cytokine_receptors & 1.74949 & 1.417618 & 2.159056 \\
\hline OXTR & Cytokine_receptors & 1.405633 & 1.201078 & 1.645026 \\
\hline PDGFC & Cytokines & 1.619596 & 1.361091 & 1.927198 \\
\hline PDGFRB & Cytokines & 1.508678 & 1.304742 & 1.744491 \\
\hline PTGER3 & Cytokine_receptors & 1.630084 & 1.371071 & 1.938027 \\
\hline RBP1 & Antimicrobials & 1.486226 & 1.289005 & 1.713621 \\
\hline SDC2 & Cytokine_receptors & 1.822409 & 1.522803 & 2.180962 \\
\hline SEMA4C & Chemokines & 2.225396 & 1.693848 & 2.92375 \\
\hline SHC4 & NaturalKiller_cell_cytotoxicity & 1.926967 & 1.615581 & 2.298371 \\
\hline SLIT2 & Chemokines & 1.469846 & 1.32759 & 1.627345 \\
\hline STC2 & Cytokines & 1.506128 & 1.26909 & 1.78744 \\
\hline TGFB2 & Cytokines & 2.056681 & 1.636983 & 2.583983 \\
\hline TGFB3 & Cytokines & 1.5918 & 1.342242 & 1.887756 \\
\hline THBS1 & Antigen_processing_and_presentation & 1.487585 & 1.310701 & 1.688341 \\
\hline TNC & Chemokines & 1.286883 & 1.174997 & 1.409424 \\
\hline TPM2 & Antimicrobials & 1.441131 & 1.297227 & 1.600998 \\
\hline
\end{tabular}

the "glmnet" R package, where the time-dependent ROC curve was plotted with "survivalROC" and the Kaplan-Meier survival curve was generated with "survminer." This analysis facilitated the evaluation of the risk score's performance [14]. In addition, with the "rms" $\mathrm{R}$ package, the prognostic signature was visualized in the form of a nomogram, and 

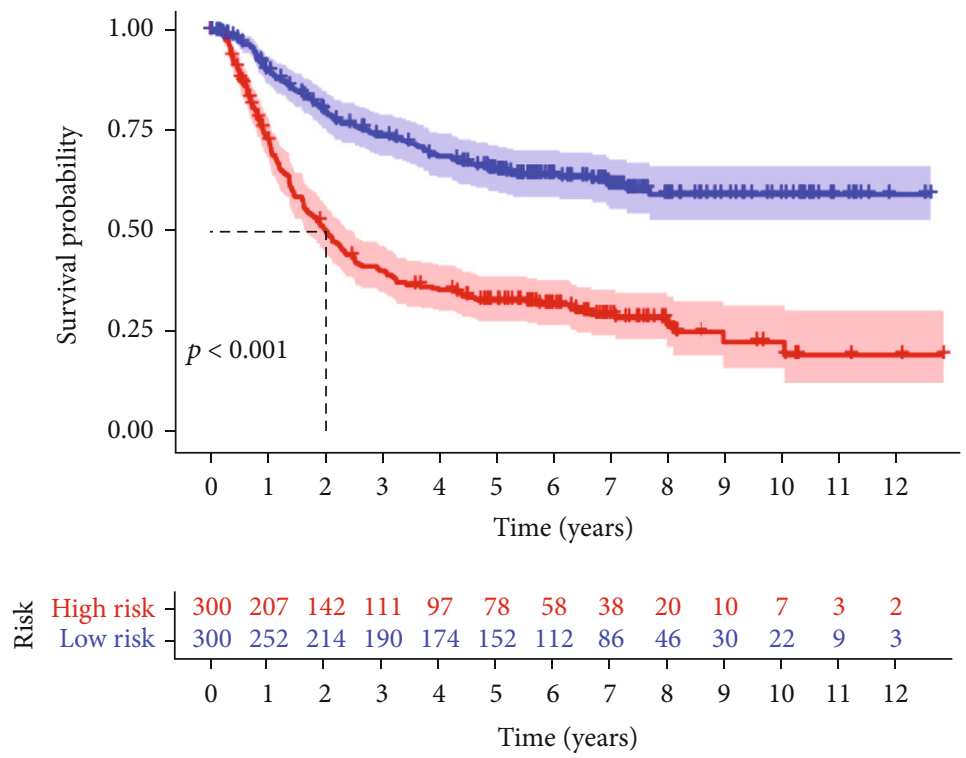

Risk

+ High risk

+ Low risk

(a)

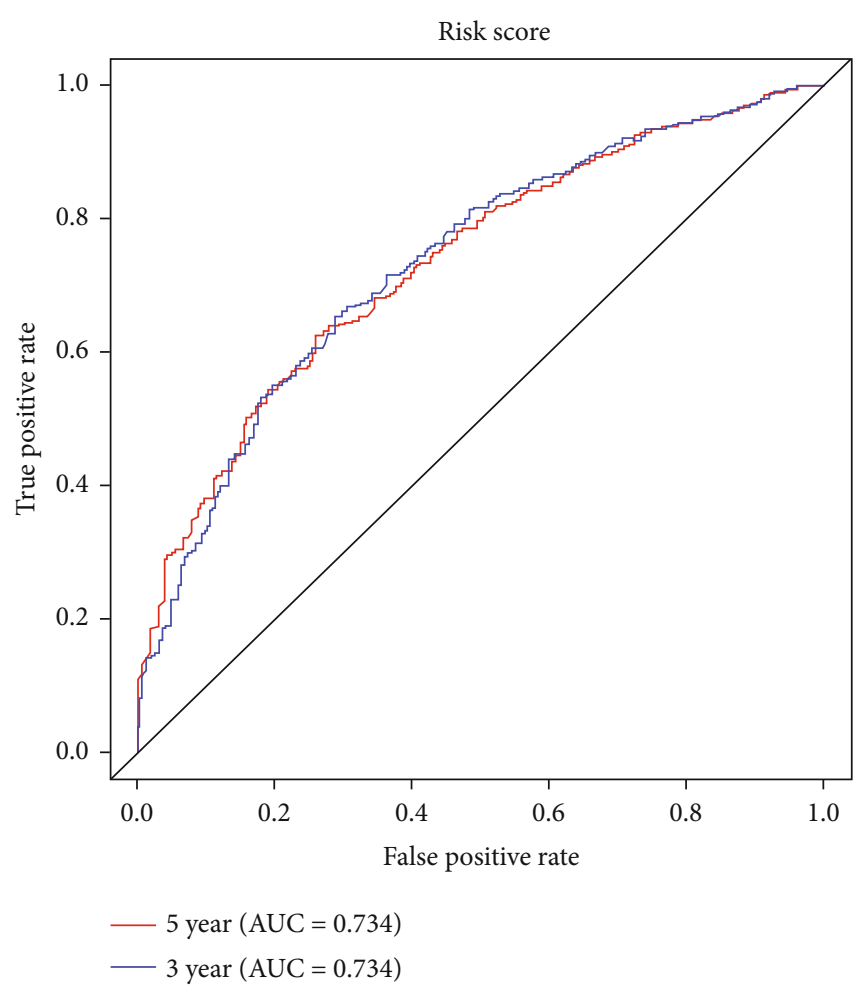

(b)

Figure 2: Continued. 


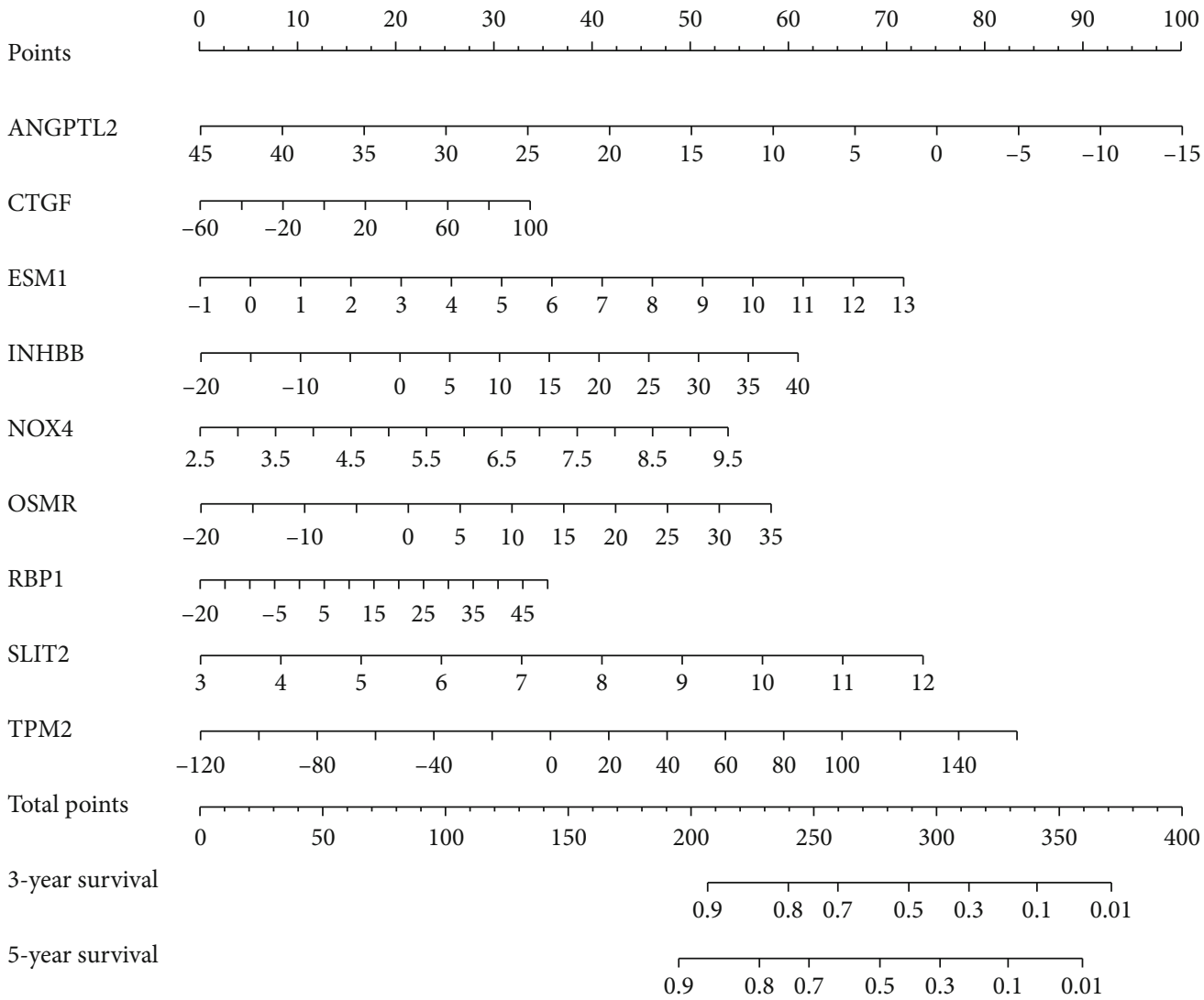

(c)
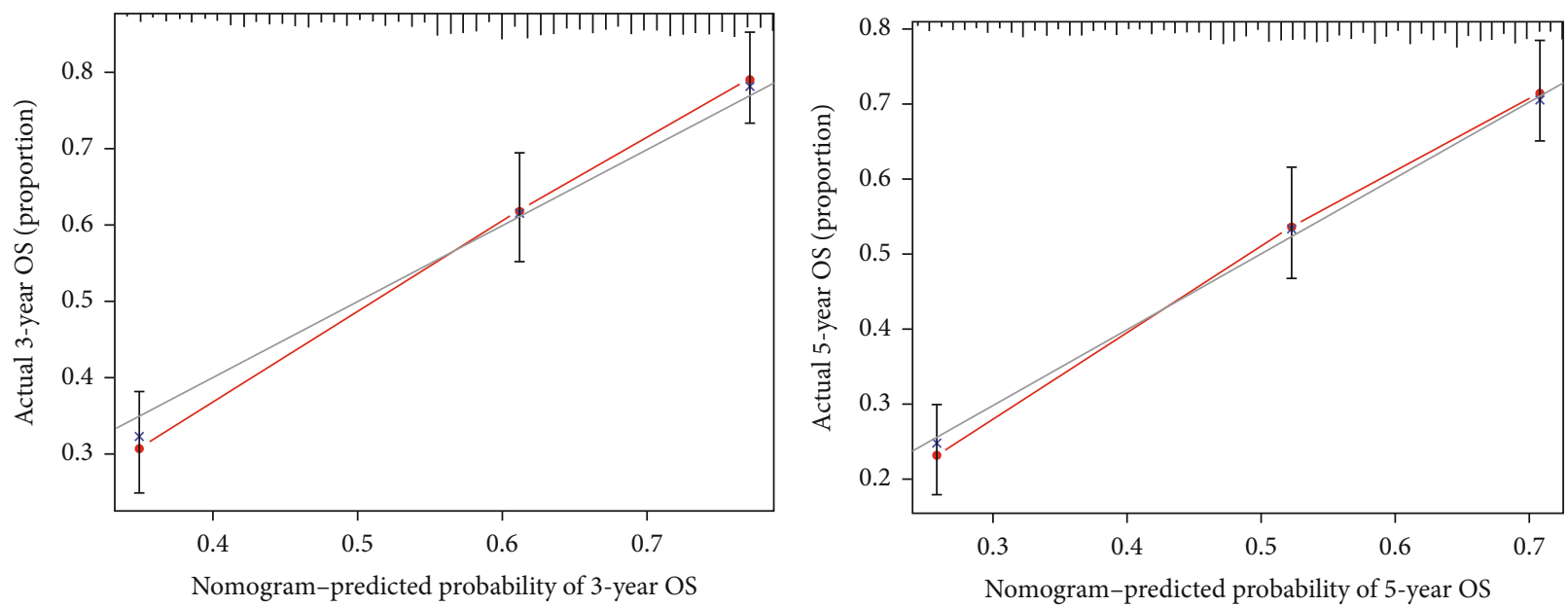

(d)

Figure 2: Construction of a nine-gene prognostic signature. $(\mathrm{a}, \mathrm{b})$ Kaplan-Meier survival analysis and time-dependent ROC analysis regarding risk score and OS in the training cohort. (c, d) Prognostic signature visualized as a nomogram and tested by the calibration curve.

the calibration curve was used to evaluate the nomogram's predictive accuracy. The prognostic signature's performance was externally validated in the three independent cohorts (GSE26253, $n=432$; GSE84437, $n=431$; and TCGA, $n=336$ ).

2.5. Analysis of the Relationship between Immune Cell Infiltration and the Prognostic Signature. Immune cell infiltration (ICI) was quantified by the CIBERSORT method, and the sample filtration threshold was $p<0.05$. We used the Wilcox test to compare the ICI difference between different risk groups, where the statistical significance was expressed as $p<0.05$.

2.6. Analysis of the Relationship between Clinical Characteristics and Prognostic Signature. We analyzed the prognostic differences among patients with GC with different clinical features 


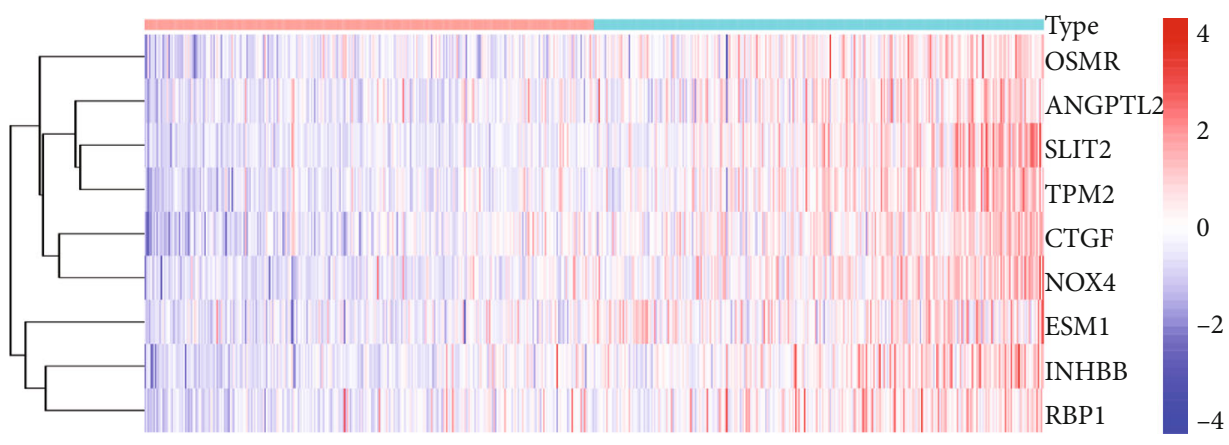

Type
High
Low

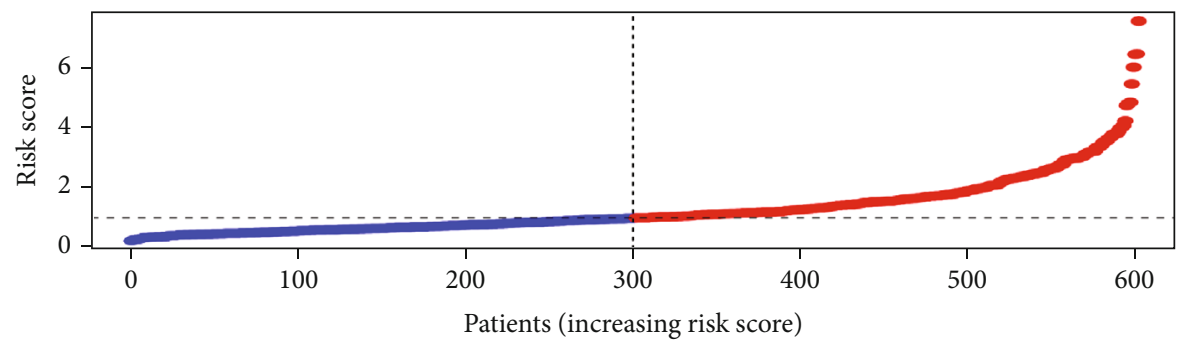

- High risk

- Low risk

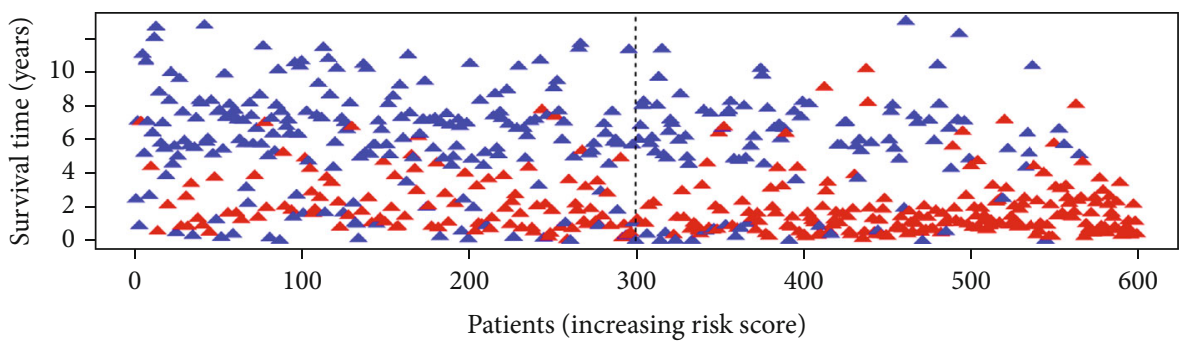

\ Dead

\ Alive

FIGURE 3: Heatmap, risk score distribution, and survival status of patients in the training cohort.

TABLE 2: Univariate and multivariate Cox regression analyses.

\begin{tabular}{|c|c|c|c|c|}
\hline Univariate Cox & & HR & HR.95L & HR.95H \\
\hline Gender & Male/female & 1.109543 & 0.870532 & 1.414176 \\
\hline Age & $>65 / \leq 65$ & 1.312168 & 1.043428 & 1.650125 \\
\hline Stage & IV/III/II/I & 2.339875 & 2.041174 & 2.682288 \\
\hline Lauren classification & Diffuse\&mixed/intestinal & 1.371609 & 1.092159 & 1.722562 \\
\hline Risk score & High/low & 2.677984 & 2.102358 & 3.411215 \\
\hline \multicolumn{5}{|l|}{ Multivariate Cox } \\
\hline Gender & Male/female & 1.015188 & 0.793251 & 1.29922 \\
\hline Age & $>65 / \leq 65$ & 1.541482 & 1.223368 & 1.942316 \\
\hline Stage & $\mathrm{IV} / \mathrm{III} / \mathrm{II} / \mathrm{I}$ & 2.284629 & 1.984801 & 2.62975 \\
\hline Lauren classification & Diffuse\&mixed/intestinal & 1.073366 & 0.85091 & 1.353981 \\
\hline Risk score & High/low & 2.370156 & 1.850191 & 3.036248 \\
\hline
\end{tabular}




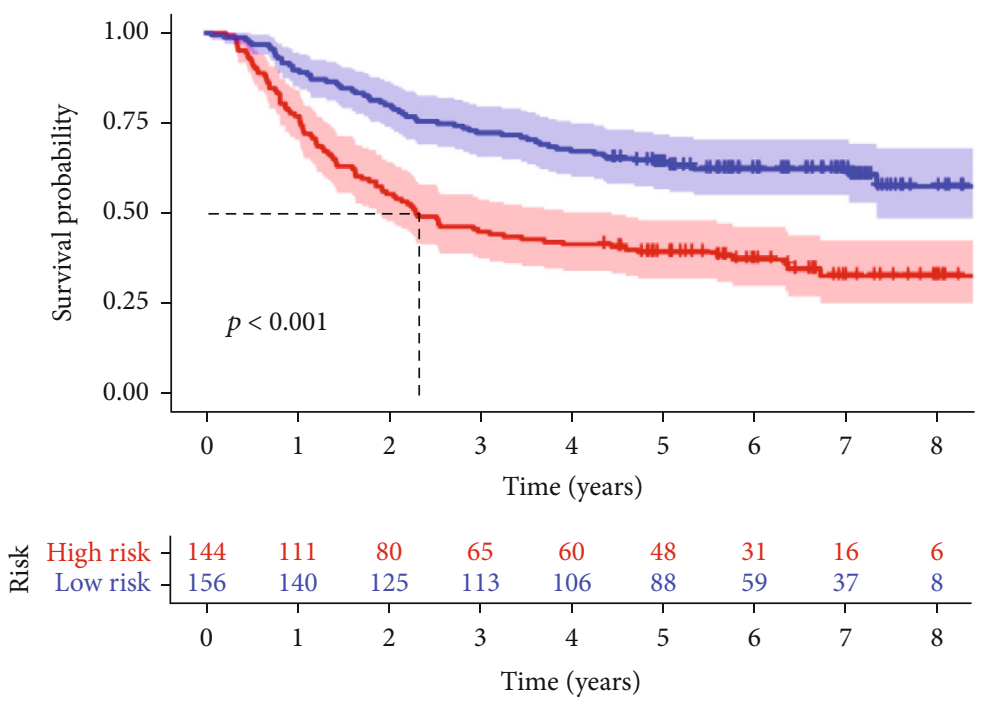

Risk

$$
\begin{aligned}
& + \text { High risk } \\
& + \text { Low risk }
\end{aligned}
$$

(a)

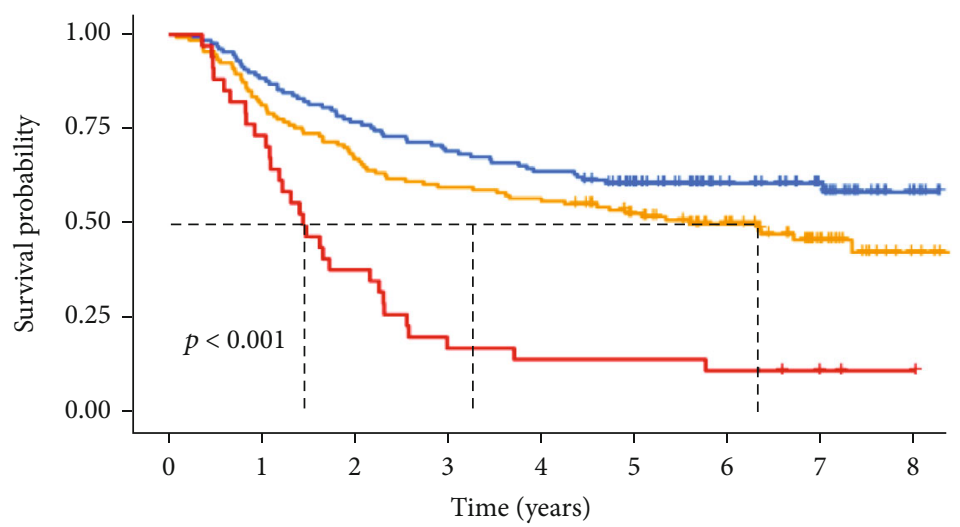

Number at risk

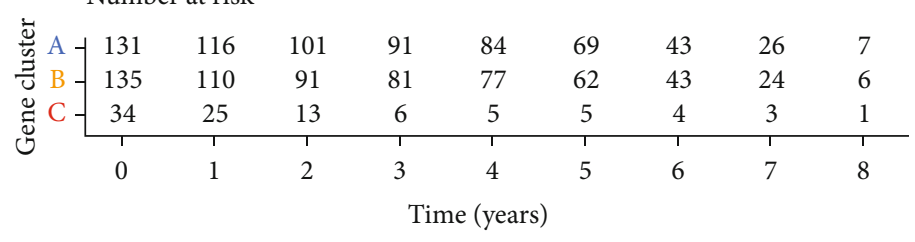

Gene cluster

$$
\begin{aligned}
& +\mathrm{A} \\
& +\mathrm{B} \\
& +\mathrm{C}
\end{aligned}
$$

(b)

Figure 4: Continued. 


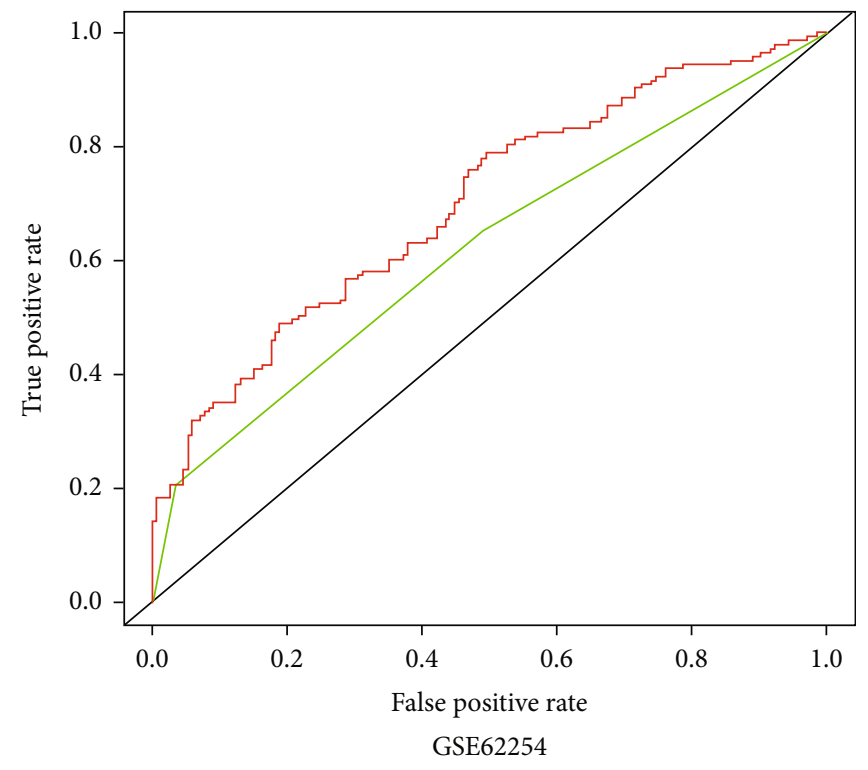

- Risk score $(\mathrm{AUC}=0.700)$

- Cluster (AUC $=0.620$ )

(c)

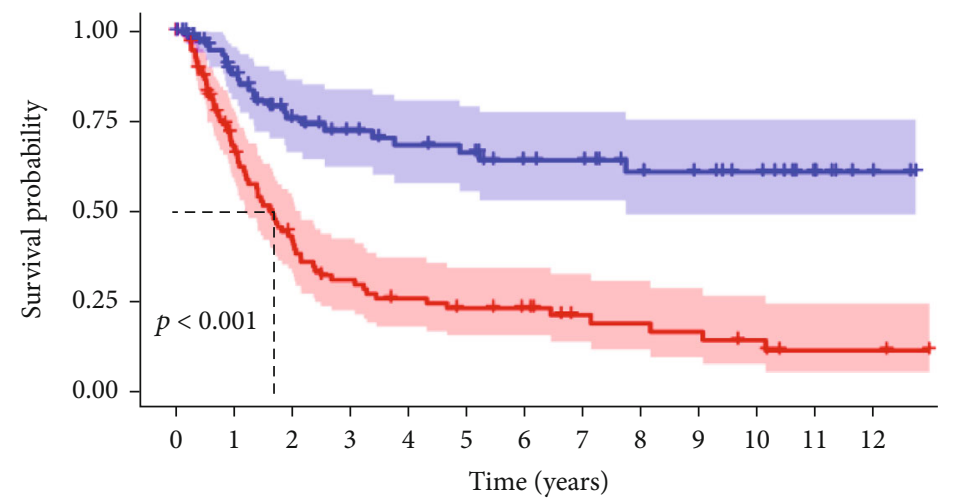

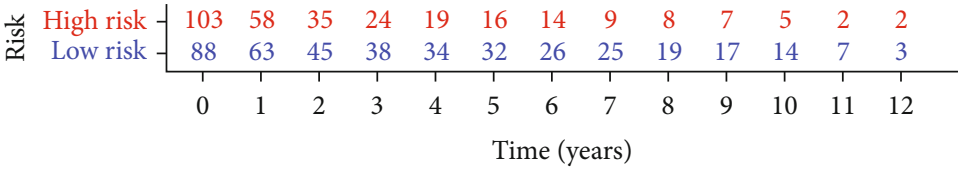

Risk

+ High risk

+ Low risk

(d)

Figure 4: Continued. 


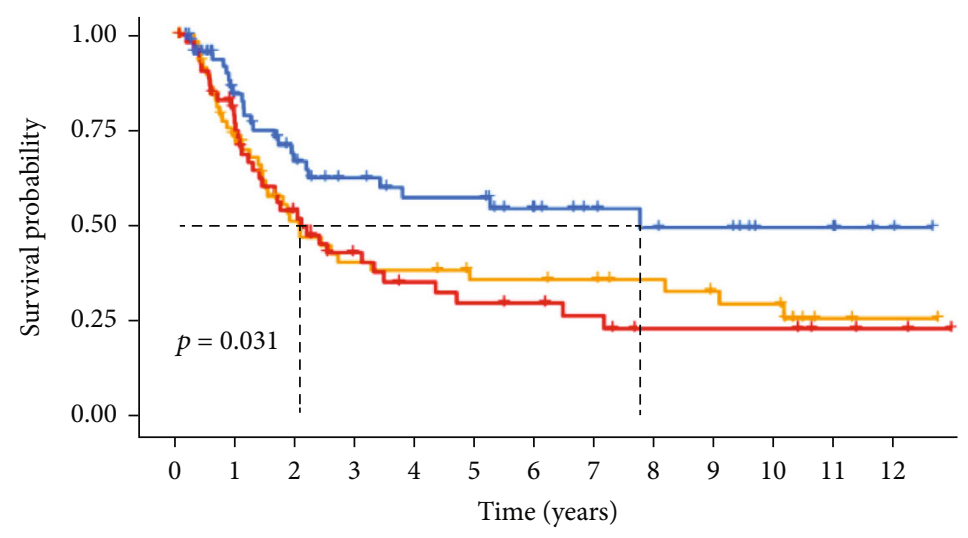

Number at risk

\begin{tabular}{|c|c|c|c|c|c|c|c|c|c|c|c|c|}
\hline 72 & 45 & 31 & 26 & 22 & 22 & 15 & 12 & 10 & 9 & 5 & 4 & 2 \\
\hline 65 & 39 & 24 & 19 & 18 & 15 & 15 & 14 & 12 & 10 & 9 & 2 & 1 \\
\hline 54 & 37 & 25 & 17 & 13 & 11 & 10 & 8 & 5 & 5 & 5 & 3 & 2 \\
\hline 0 & 1 & 2 & 3 & 4 & 5 & 6 & 7 & 8 & 9 & 10 & 11 & 12 \\
\hline
\end{tabular}

Gene cluster

$+\mathrm{A}$

$+\mathrm{B}$

$+\mathrm{C}$

(e)

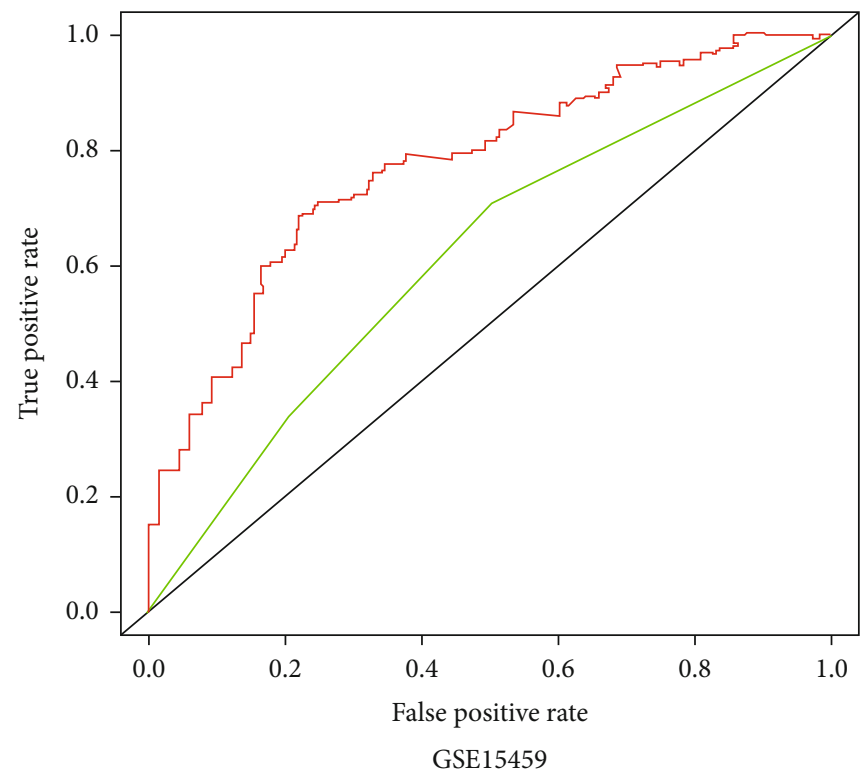

— Risk score $(\mathrm{AUC}=0.772)$

- Cluster $(\mathrm{AUC}=0.616)$

(f)

Figure 4: Continued. 


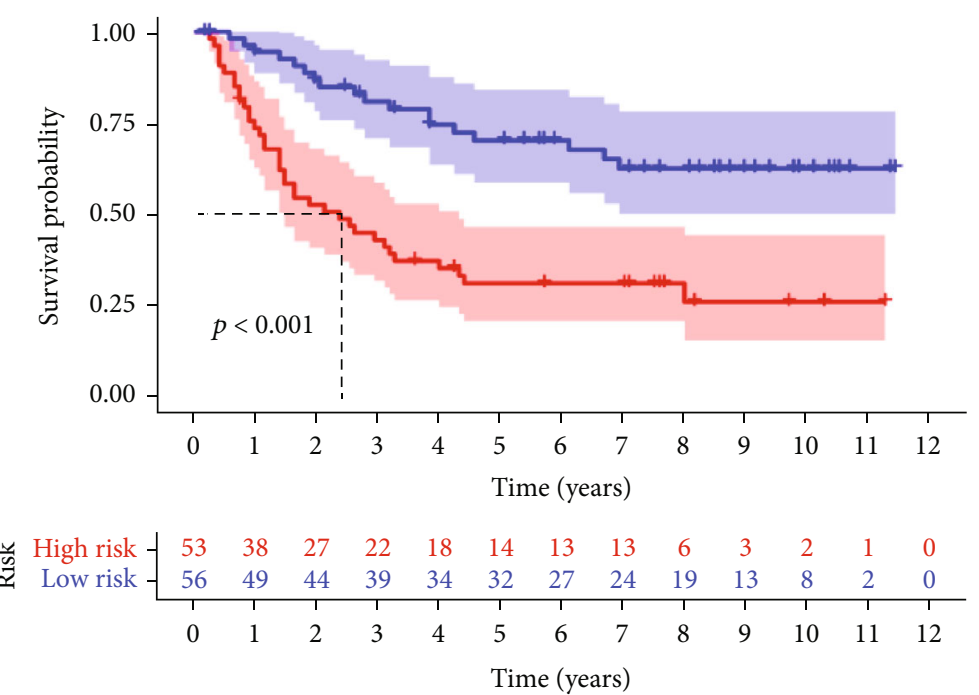

Risk

+ High risk

+ Low risk

(g)

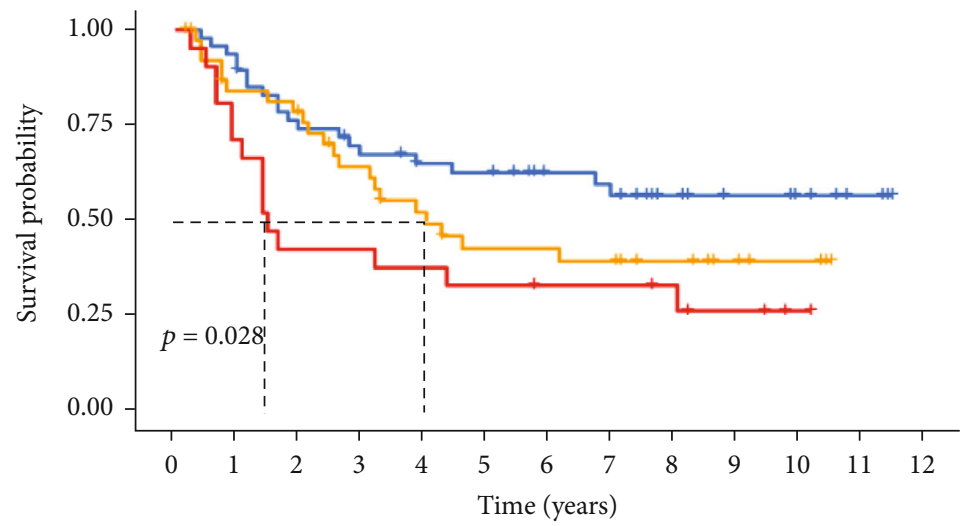

Number at risk

\begin{tabular}{|c|c|c|c|c|c|c|c|c|c|c|c|c|}
\hline$A-48$ & 41 & 34 & 30 & 27 & 26 & 21 & 19 & 12 & 8 & 6 & 3 & 0 \\
\hline$B-40$ & 31 & 28 & 22 & 17 & 13 & 13 & 12 & 8 & 5 & 3 & 0 & 0 \\
\hline 21 & 15 & 9 & 9 & 8 & 7 & 3 & 6 & 5 & 3 & 1 & 0 & 0 \\
\hline 0 & 1 & 2 & 3 & 4 & 5 & 6 & 7 & 8 & 9 & 10 & 11 & 12 \\
\hline
\end{tabular}

Gene cluster

$+\mathrm{A}$
$+\mathrm{B}$
$+\mathrm{C}$

(h)

FIgure 4: Continued. 


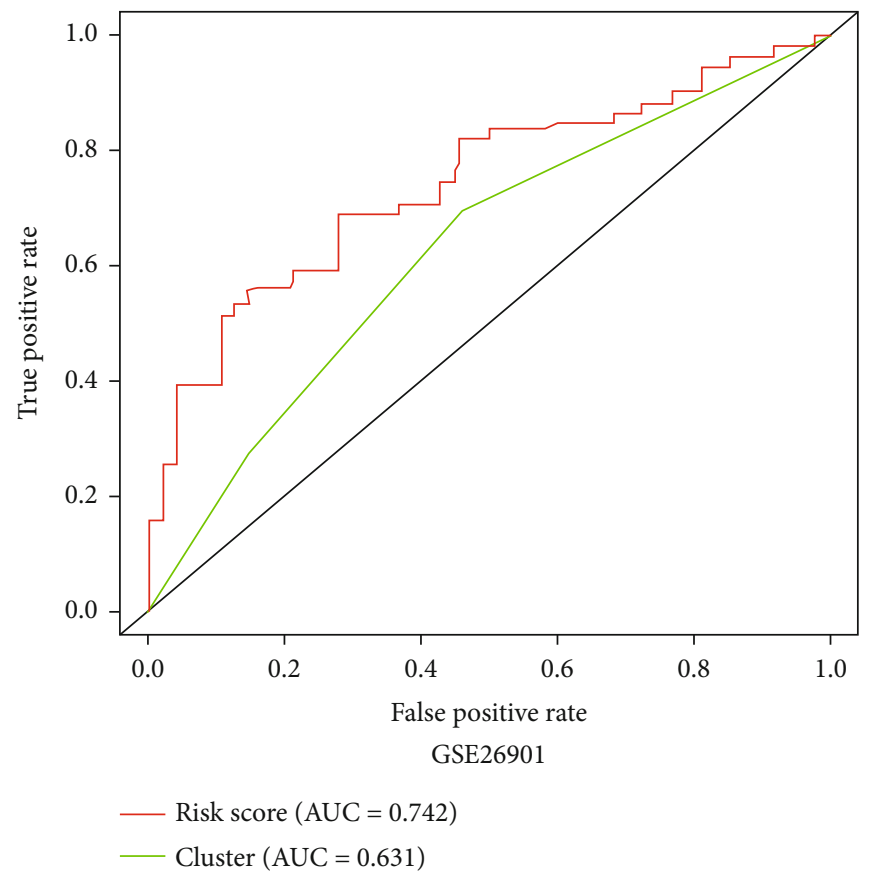

(i)

FIGURE 4: Internal validation of the prognostic signature. $(\mathrm{a}-\mathrm{c})$ Kaplan-Meier survival analysis and the time-dependent ROC analysis of the risk score and gene cluster for predicting OS in the GSE62254 cohort. (d-f) Kaplan-Meier survival analysis and the time-dependent ROC analysis of the risk score and gene cluster for predicting OS in the GSE15459 cohort. (g-i) Kaplan-Meier survival analysis and the timedependent ROC analysis of the risk score and gene cluster for predicting OS in the GSE26901 cohort.

and conducted a comparison of the low- and high-risk groups' clinical characteristics. In addition, we assessed the distribution of risk scores of patients with different clinical features.

2.7. Comparison of the Immune Response in Different Risk Groups. To reveal the potential immune signature of the prognostic model, we selected 93 immune-related gene sets from "c5.all.v7.3.symbols.gmt" (downloaded from http:// www.gsea-msigdb.org/gsea) and conducted a gene set enrichment analysis (GSEA) of the different risk groups. In general, the higher the enrichment score (ES), the stronger the activity of the pathway.

\subsection{Analysis of the Relationship between Tumor} Microenvironment and Risk Score. The stromal cells and immune cells were main components in the tumor microenvironment (TME). The ESTIMATE (Estimation of STromal and Immune cells in MAlignant Tumor tissues using Expression data) algorithm could quantify the immune and stromal components in the tumor microenvironment (TME) by analyzing the specific gene expression characteristics of immune and stromal cells, which was employed to calculate the Stromal Score (captures the presence of stroma in tumor tissue), Immune Score (represents the infiltration of immune cells in tumor tissue), and ESTIMATE Score (equaled the sum of Stromal Score and Immune Score) [18]. We assigned patients into high- and low-level groups by comparison to the median value of the scores and performed Kaplan-Meier survival analysis to investigate the relationship between the scores and the GC prognosis. The Spearman correlation test was used to analyze the association of the risk score with the TME scores.

\section{Results}

3.1. Patients with GC Had Different Clinical Outcomes with Different Gene Clusters. A total of 45 overlapping prognosisrelated genes were identified in the three independent cohorts (GSE62254, GSE15459, and GSE26901) (Figure 1(a), Table 1). The 600 patients with GC were divided into three subtypes based on the 45 gene cluster analysis (Figure 1(b)). There were significant differences in the OS among patients with different subtypes of GC (Figure 1(c)), which preliminarily implied the significant impact of the expression of the 45 genes on the prognosis of GC.

3.2. A Nine-Gene Prognostic Signature Was Established in the Training Cohort. The risk score established by LASSO regression and multivariate Cox regression analyses was calculated as follows: ANGPTL2 $*-0.03827+$ CTGF $* 0.004835+$ ESM $1 * 0.117437+\mathrm{INHBB} * 0.0233+\mathrm{NOX} 4 * 0.17667+$ OSMR $* 0.02425+\mathrm{RBP} 1 * 0.011599+\mathrm{SLIT} 2 * 0.18834+\mathrm{TPM} 2 *$ 0.006843 . The 600 patients with GC were divided into lowand high-risk groups according to the median risk score (0.966). The high-risk group had a lower OS than the lowrisk group (Figure 2(a)). The area under the curve (AUC) values for the risk score predicting 3- and 5-year OS were 0.734 and 0.734 , respectively (Figure 2(b)). The calibration curve also revealed the consistency between the actual OS and the signature-predicted OS (Figures 2(c) and 2(d)). The 


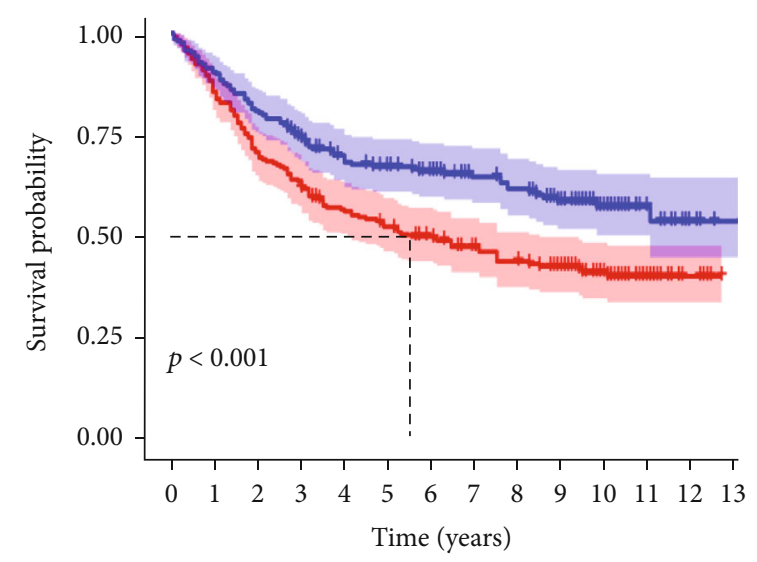

뜨 High risk - $\begin{array}{llllllllllllll}222 & 190 & 157 & 141 & 120 & 110 & 99 & 81 & 73 & 65 & 47 & 26 & 8 & 0\end{array}$

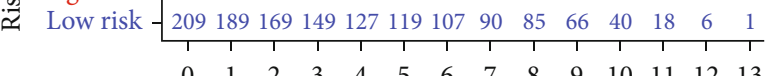
Time (years)

$$
\begin{aligned}
& \text { Risk } \\
& + \text { High risk } \\
& + \text { Low risk }
\end{aligned}
$$

(a)
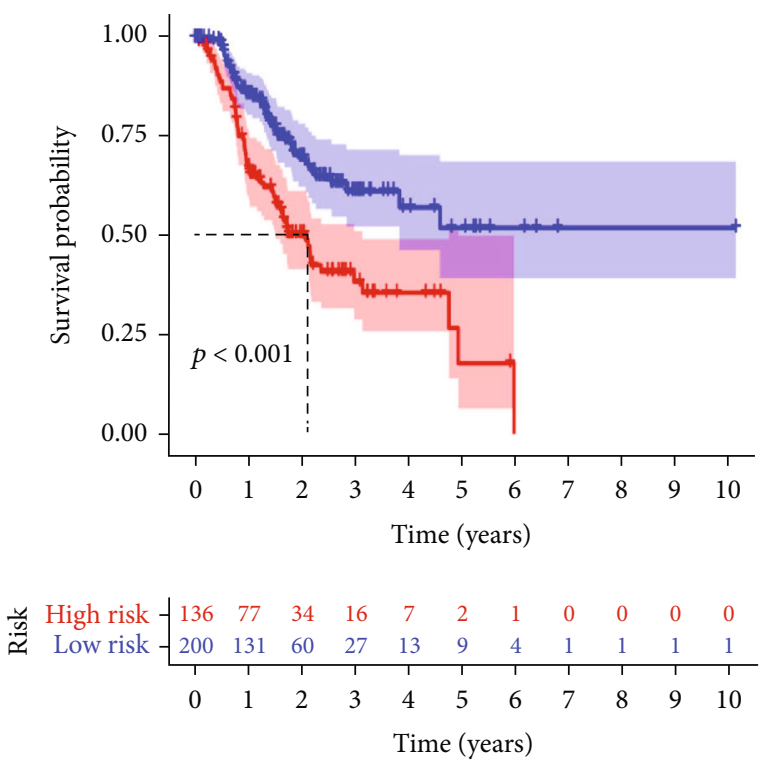

Risk

+ High risk

+ Low risk

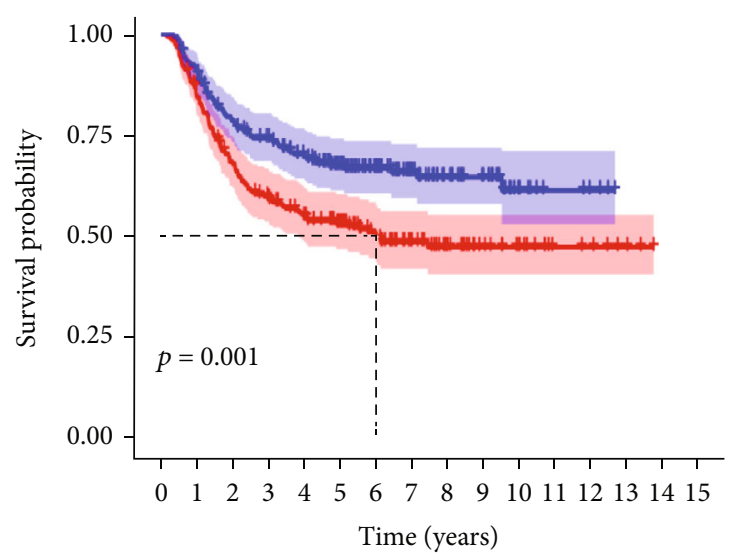

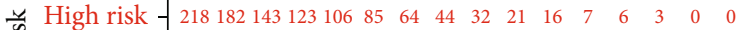

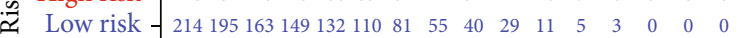
$\begin{array}{llllllllllllllll}0 & 1 & 2 & 3 & 4 & 5 & 6 & 7 & 8 & 9 & 10 & 11 & 12 & 13 & 14 & 15\end{array}$ Time (years)

Risk

+ High risk

+ Low risk

(b)

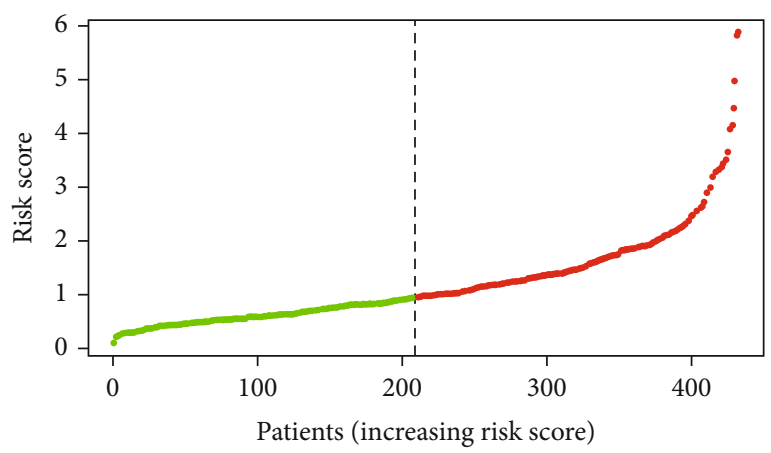

- High risk

- Low risk

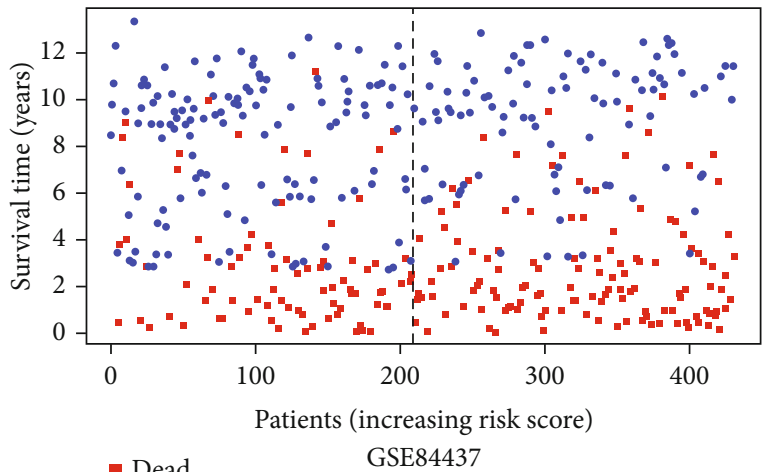

- Alive

(c)

(d)

Figure 5: Continued. 


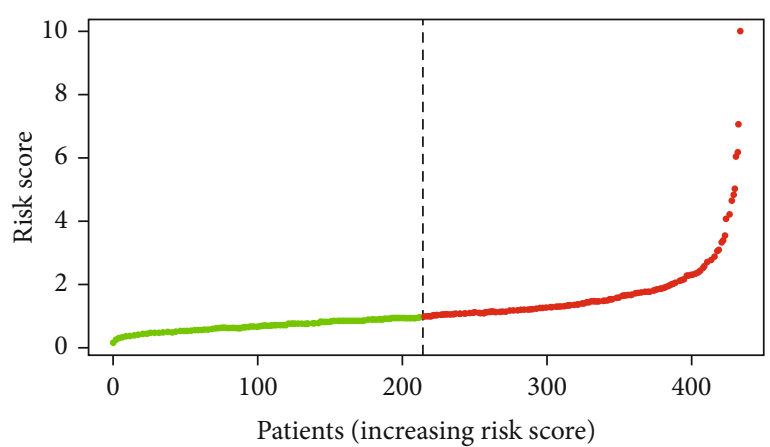

- High risk

- Low risk

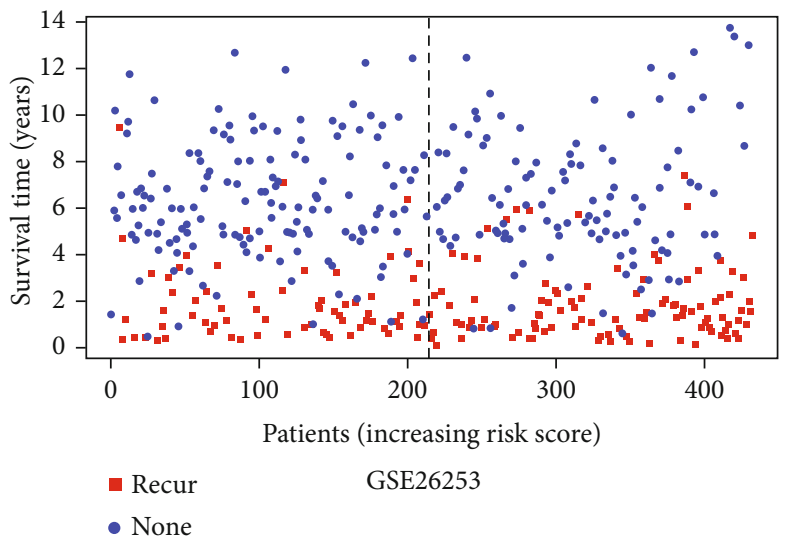

(e)

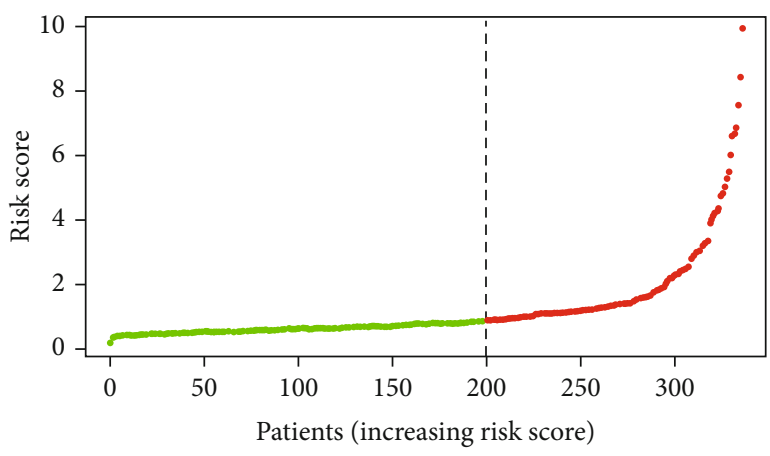

- High risk

- Low risk

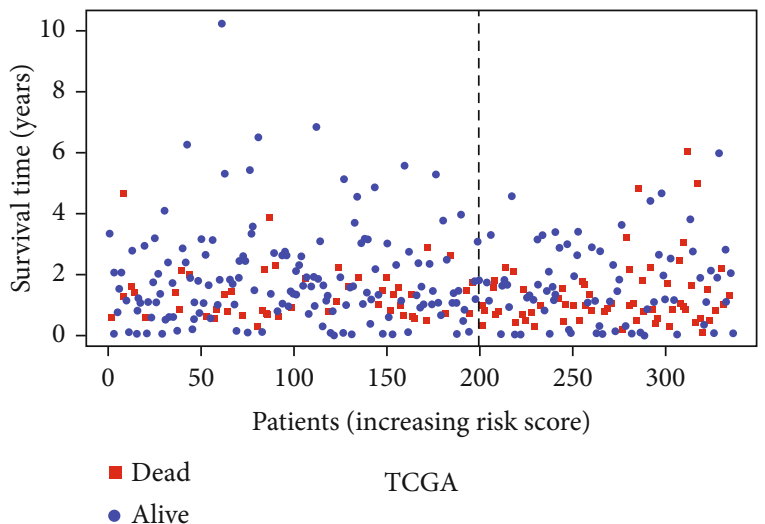

(f)

FIgURE 5: External validation of the prognostic signature. $(\mathrm{a}-\mathrm{c})$ Kaplan-Meier survival analysis of the prognostic signature for predicting OS in the GSE84437, GSE26253, and TCGA cohorts. (d-f) The risk score distribution and the survival status of patients in the GSE84437, GSE26253, and TCGA cohorts.

high-risk group presented upregulated expression levels of the 9 genes, and the patients' risk of death increased as the risk score increased (Figure 3). Univariate and multivariate Cox regression identified the risk score as an independent indicator for the prognosis of patients with GC (Table 2), and the detailed clinical information is displayed in supplement material 1.

3.3. The Prognostic Signature Showed High Accuracy for the Prediction of the OS of Patients with GC in the GSE62254, GSE15459, and GSE26901 Cohorts. The high-risk group had a significantly lower OS than the low-risk group in each independent cohort (Figures 4(a), 4(d), and 4(g)). The accuracy of the risk score was higher than that of the gene cluster for the stratification of the prognostic risk of patients with GC (Figures 4(b), 4(c), 4(e), 4(f), 4(h), and 4(i)).

3.4. The Prognostic Signature Showed Robustness through External Validation. Consistent with the results of internal validation, compared with the low-risk group in each independent cohort, the high-risk group had significantly reduced OS and recurrence-free survival (RFS) (Figures 5(a)-5(c)). As the risk score increased, so did the risk for death and recurrence (Figures 5(d)-5(f)).
3.5. The High- and Low-Risk Groups Exhibited Differences in Immune Cell Infiltration. The tissues from patients with high-risk GC exhibited a lower infiltration level of resting NK cells, activated memory CD4 T cells, and activated dendritic cells. However, the tissues from patients with low-risk GC had a lower infiltration level of M2 macrophages than the high-risk group (Figures 6(a) and 6(b)). The prognosis of patients with GC with a higher infiltration level of M2 macrophages was poor; patients with a higher infiltration level of resting NK cells, activated memory CD4 T cells, and activated dendritic cells had a better prognosis (Figure 6(c)).

3.6. The Risk Score Was Closely Related to the Clinicopathological Features. The prognosis of patients with the Lauren classification intestinal type of GC was better than that of patients with the diffuse type and mixed type (Figure $7(\mathrm{a})$ ). The high-risk group had a lower proportion of patients with the intestinal type than the low-risk group (Figure 7(b)); patients with the intestinal type had a significantly lower risk score compared to those with the diffuse type and mixed type (Figure $7(\mathrm{c})$ ). The OS of patients decreased significantly with increasing disease stage (Figure $7(\mathrm{~d})$ ). The high-risk group had a significantly lower proportion of earlystage patients than the low-risk group, but almost double the 


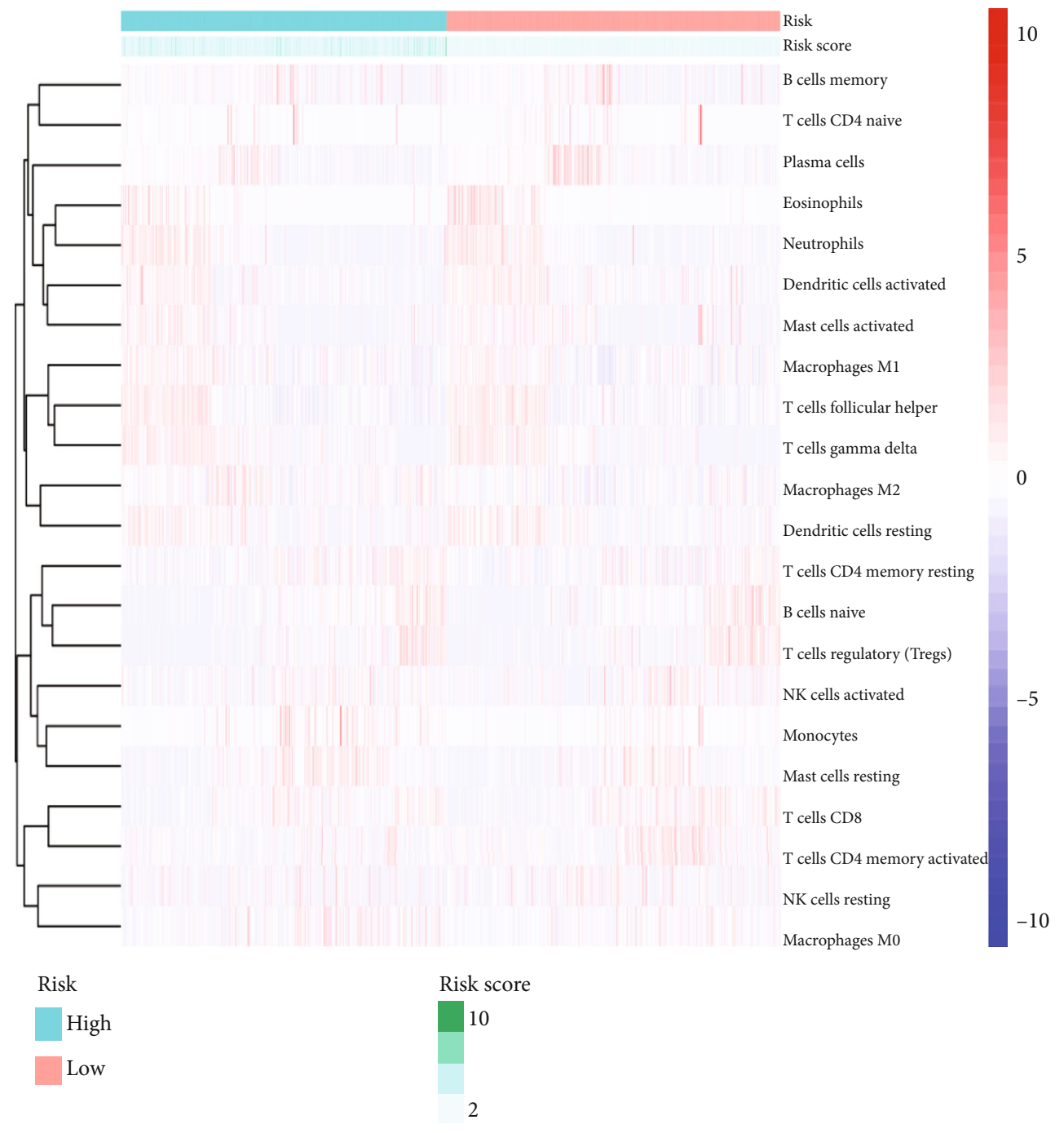

(a)

Figure 6: Continued. 


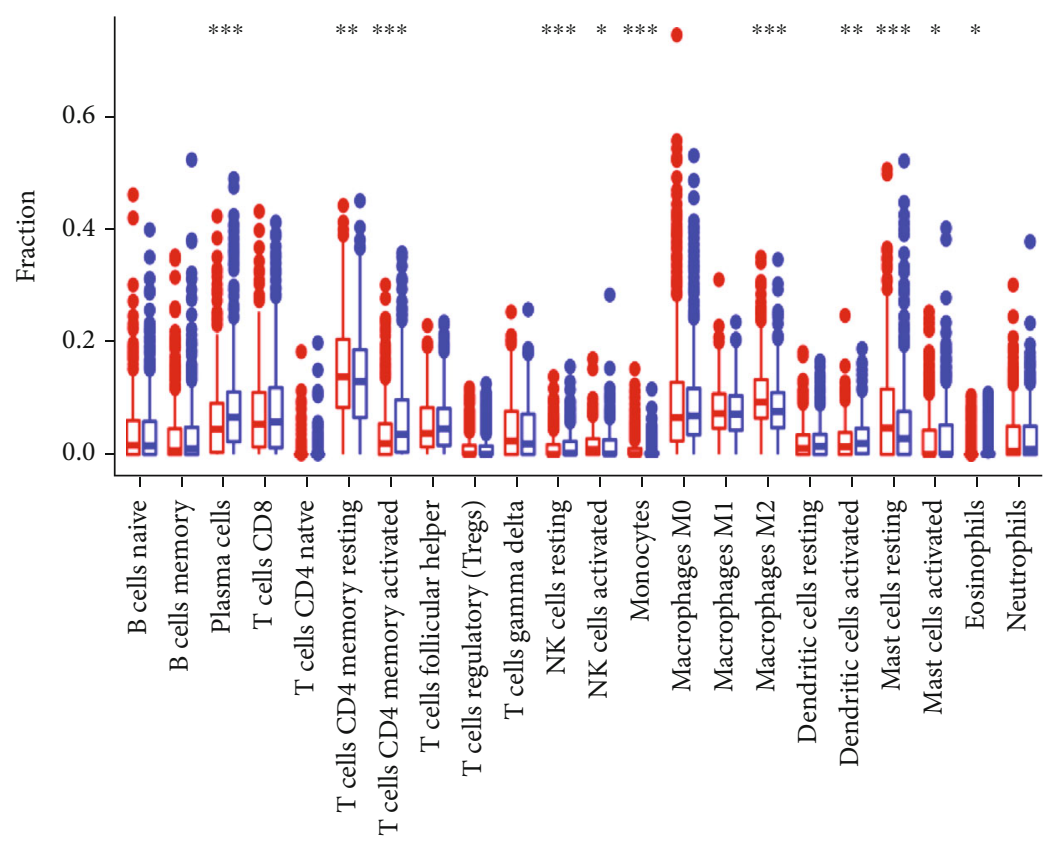

Type

申 High risk

兆 Low risk

(b)
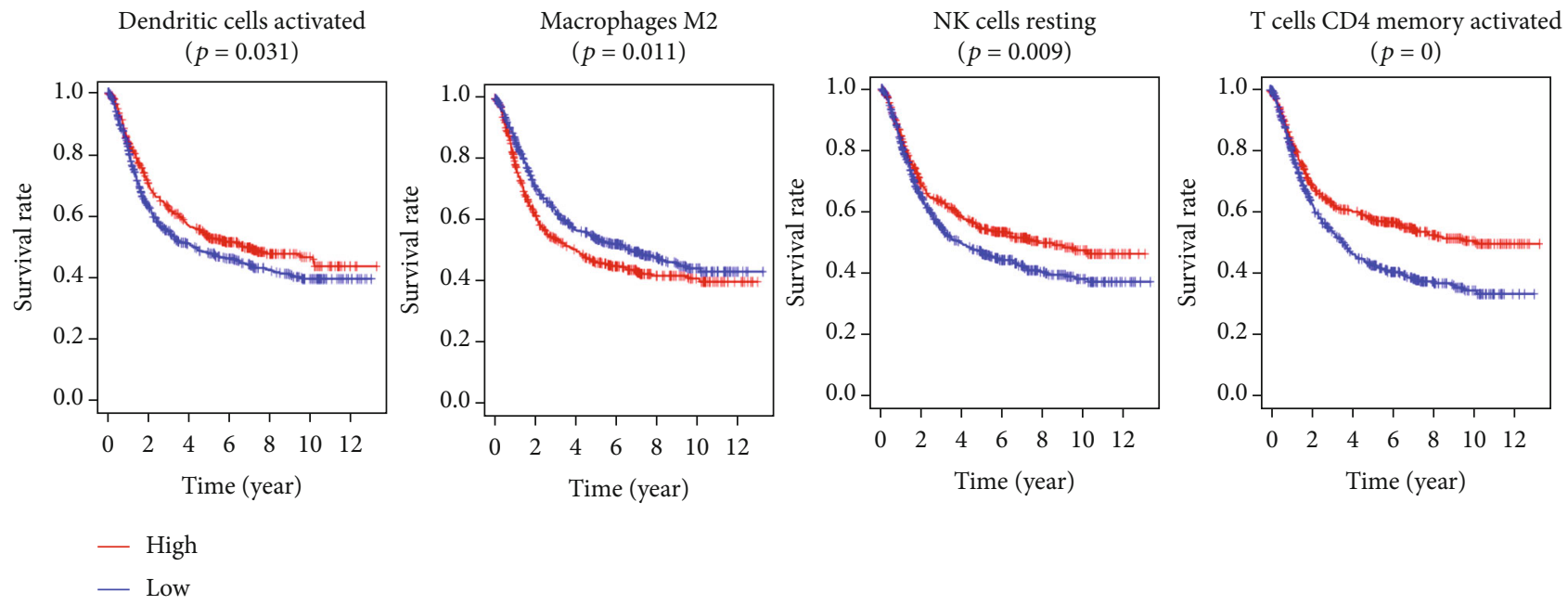

(c)

FIgURE 6: The immune cell infiltration landscape of all included samples. $(a, b)$ Heatmap and the boxplot showing the difference in immune cell infiltration in different risk groups. (c) Kaplan-Meier survival analysis regarding immune cell infiltration and OS.

number of patients in stage IV (Figure 7(e)), in line with the positive correlation between the risk score and disease stage (Figure 7(f)).

3.7. The Immune Response of the Low-Risk Group Was Stronger Than That of the High-Risk Group. In the six independent cohorts, we found that immune response-related pathway ESs in the high-risk group were lower than those in the low-risk group, including the activation of the innate immune response, regulation of the innate immune response, and immune response to tumor cells (Figure 8).
3.8. The Risk Score Exhibited a Significant Positive Correlation with the Stromal Score. We calculated the Stromal Score, Immune Score, and ESTIMATE Score of the GC samples with the ESTIMATE algorithm to facilitate the quantification of immune and stromal components in the TME in the six independent cohorts (Figure 9). Compared with the GC patients with lower Stromal Score, we found the GC patients with higher Stromal Score had a significantly decreased OS and RFS in the six independent cohorts (Figure 10). The Spearman correlation analysis suggested that the risk score and the Stromal Score were significantly 

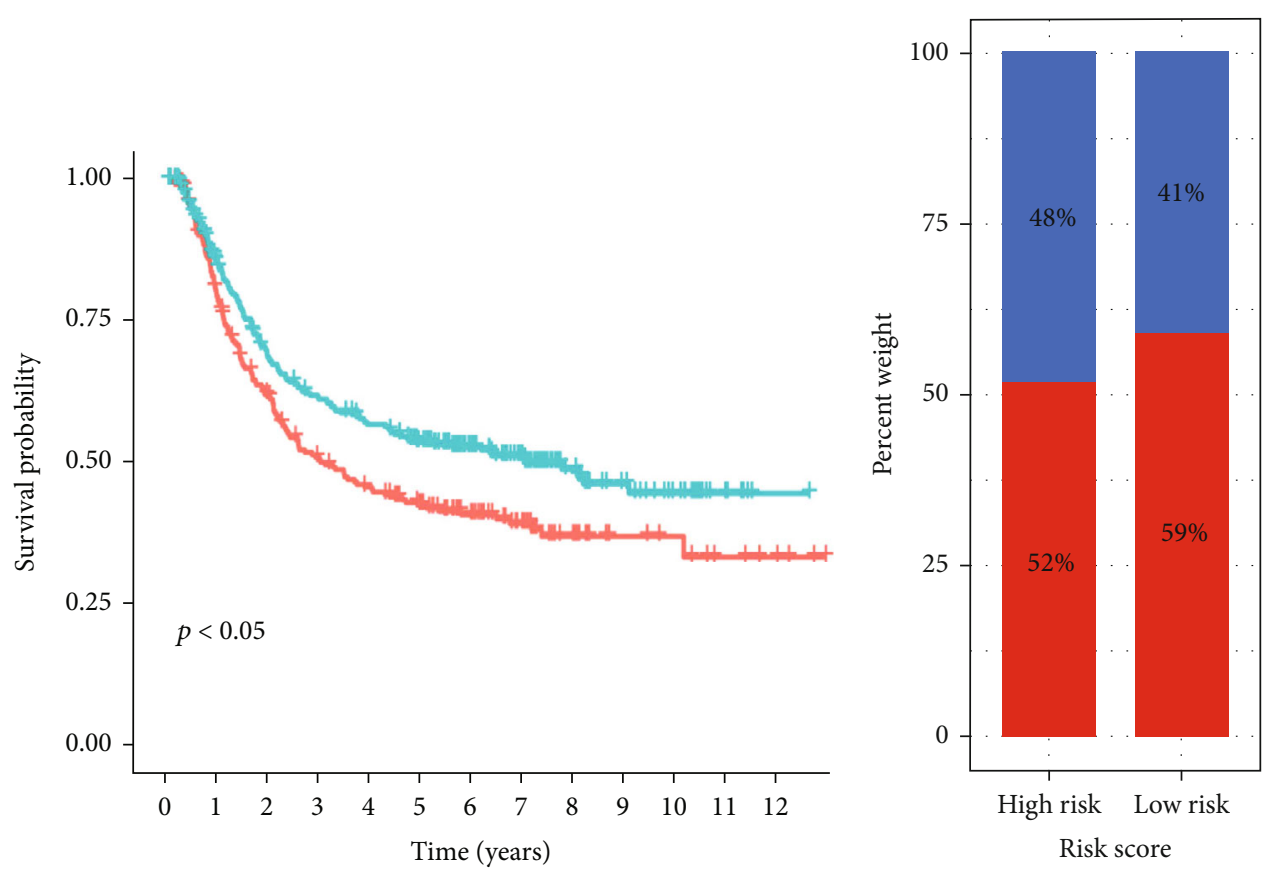

$$
\begin{aligned}
& \text { Lauren } \\
& +- \text { Diffuse\&mixed } \\
& + \text { Intestinal }
\end{aligned}
$$$$
\text { Lauren }
$$

Diffuse\&mixed

Intestinal

(a)
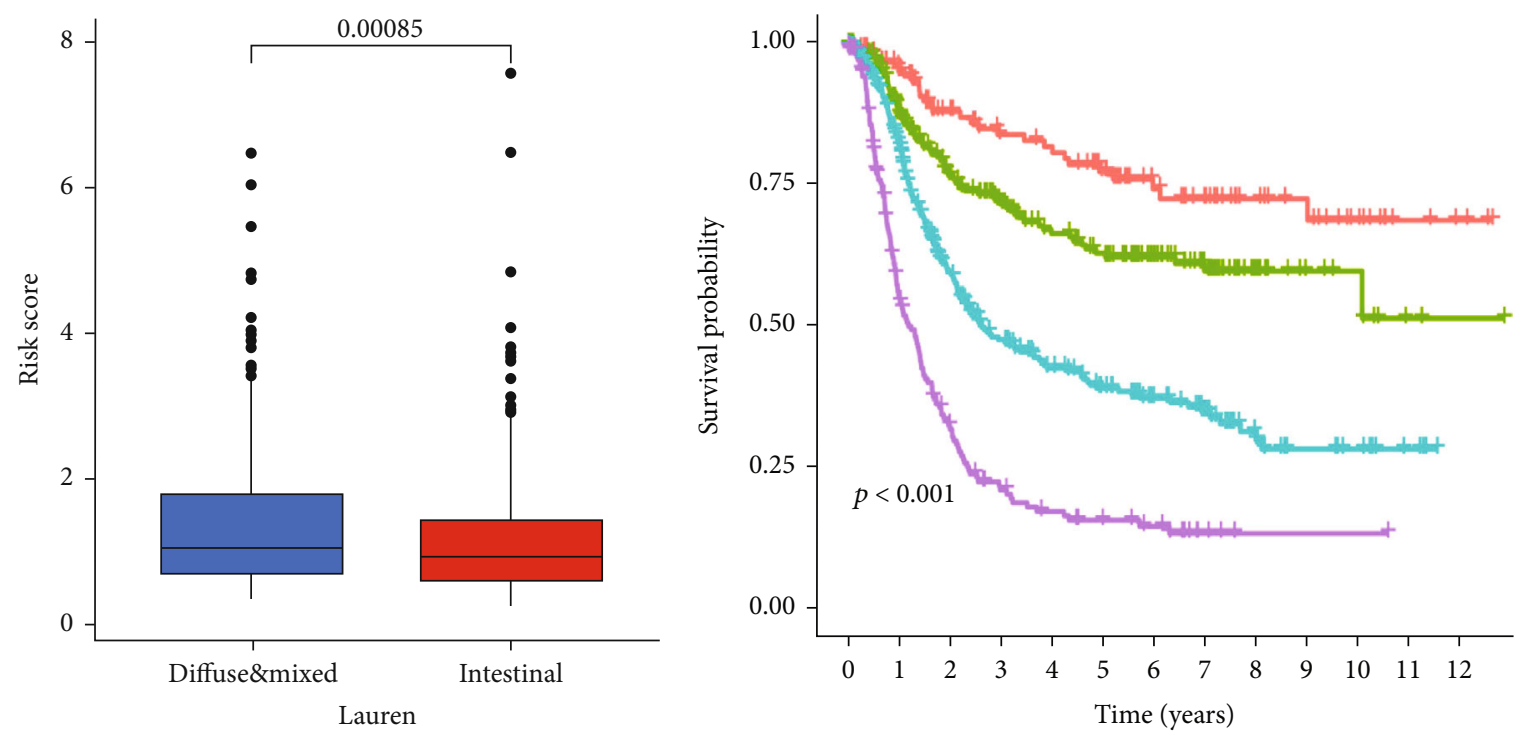

Lauren

Diffuse\&mixed

官 Intestinal

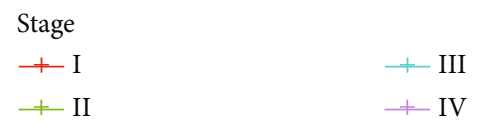

(c)

(d)

Figure 7: Continued. 


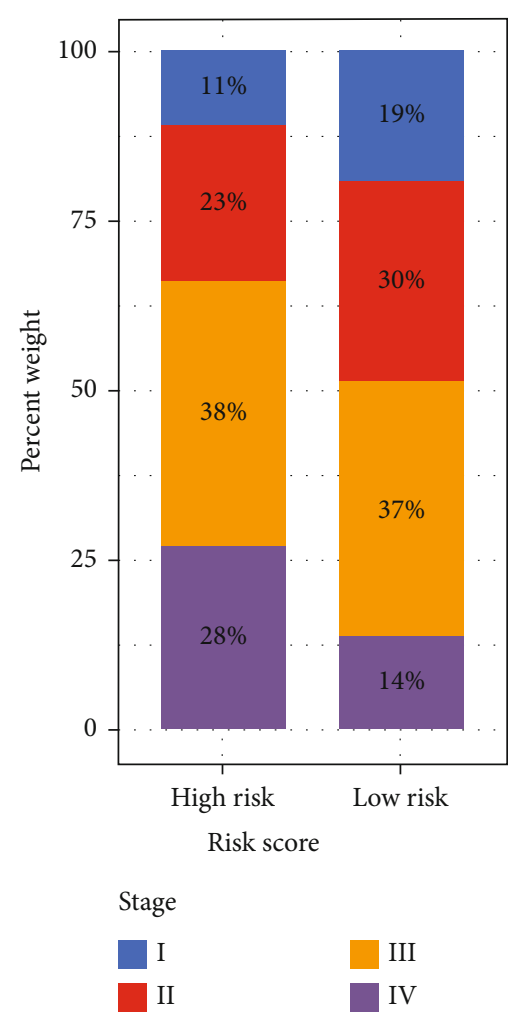

(e)
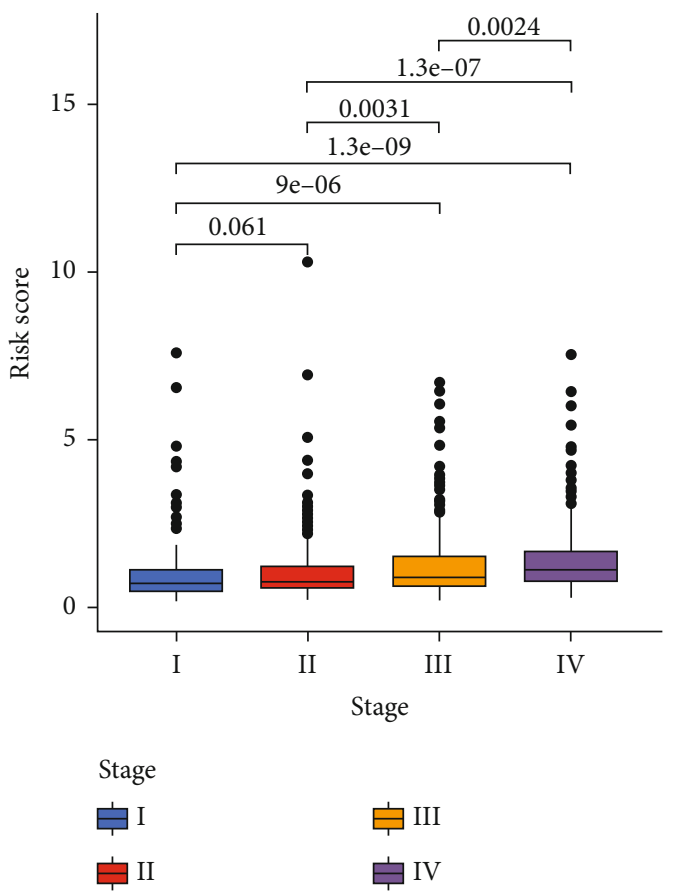

(f)

FIGURE 7: The correlation analysis between the risk score and clinical characteristics. (a) The Kaplan-Meier survival analysis regarding Lauren classification and OS. (b) Barplot of proportions of different Lauren classification subtypes in high- and low-risk groups. (c) Boxplot of the risk score difference in different Lauren classification subtypes. (d) Kaplan-Meier survival analysis regarding stage and OS. (e) Barplot of proportions of different stages in high- and low-risk groups. (f) Boxplot of the risk score difference in different stages.

positively correlated with each other (Figure 11). These results may indicate that the higher the risk score, the higher the content of neovascularization, endothelial cells, and mesenchymal stem cells around the tumor, facilitating the formation of TME to promote tumor proliferation and metastasis.

\section{Discussion}

Gastric cancer (GC) is a prevailing malignant tumor and, second to lung cancer, has the highest incidence and mortality rates in the world [19]. Most patients with GC have advanced-stage disease when they are diagnosed, which leads to poor prognosis after surgery [20]. However, it is difficult to accurately evaluate the prognosis of GC, namely, the risk for recurrence and metastasis and likely survival time, using conventional TNM staging [21]. Therefore, identifying an effective prognosis evaluation scheme for GC remains a relevant and challenging research topic.

Normally, the immune system can recognize and remove tumor cells from the tumor microenvironment [22]. However, to survive and grow, tumors use multiple mechanisms to develop immune tolerance and prevent the immune system from effectively recognizing and killing tumor cells [23]. Immune-related genes are crucial for the regulation of tumor progression and the human immune response. It is particularly important to identify how immune-related genes affect the prognosis of GC to develop a prognostic evaluation scheme for GC.

We identified 45 common prognostic immune-related genes in three different independent cohorts. According to the cluster analysis of the 45 overlapping genes, there were significant differences in the OS among different patients with different subtypes of GC. We integrated the GSE62254, GSE15459, and GSE26901 cohorts into a training cohort, screened the above 45 intersecting genes again by LASSO and multivariate Cox regression analyses, and finally created a risk score comprising 9 genes. Compared to gene cluster analysis, the risk score reduced the number of genes that need to be sequenced, saved costs, and had higher accuracy. The risk score showed universal applicability via internal and external validation. The risk score is also an independent prognostic indicator of the risk for death and recurrence in patients with GC. Additionally, we found that the risk score showed better performance than that of a previous study [24] in predicting RFS and OS in patients with GC (supplemental material 2), especially for the GSE62254 [25], GSE26253 [26], and GSE26901 [26] cohorts. Considering that the above three datasets mainly originated from Asian populations, we speculated that our model is superior in evaluating the prognosis of Asian patients with GC. To test our hypothesis, we observed the survival of Asian patients in TCGA dataset. Although only 69 patients were included, we 

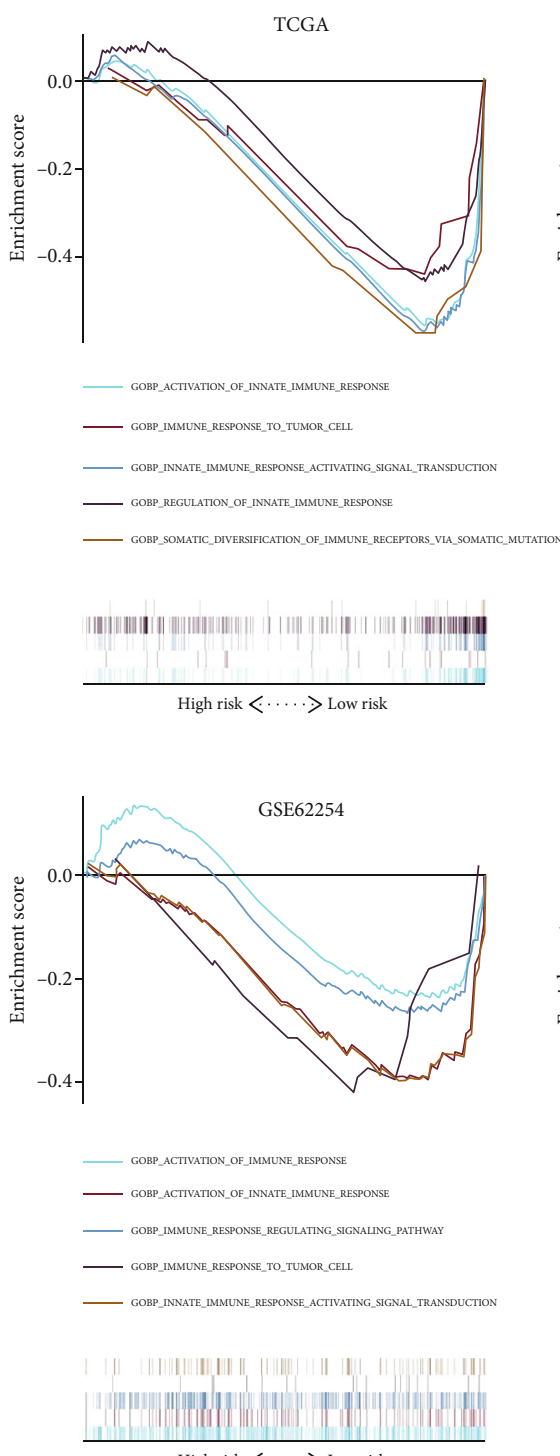

High risk $<\cdots \cdots\rangle$ Low risk
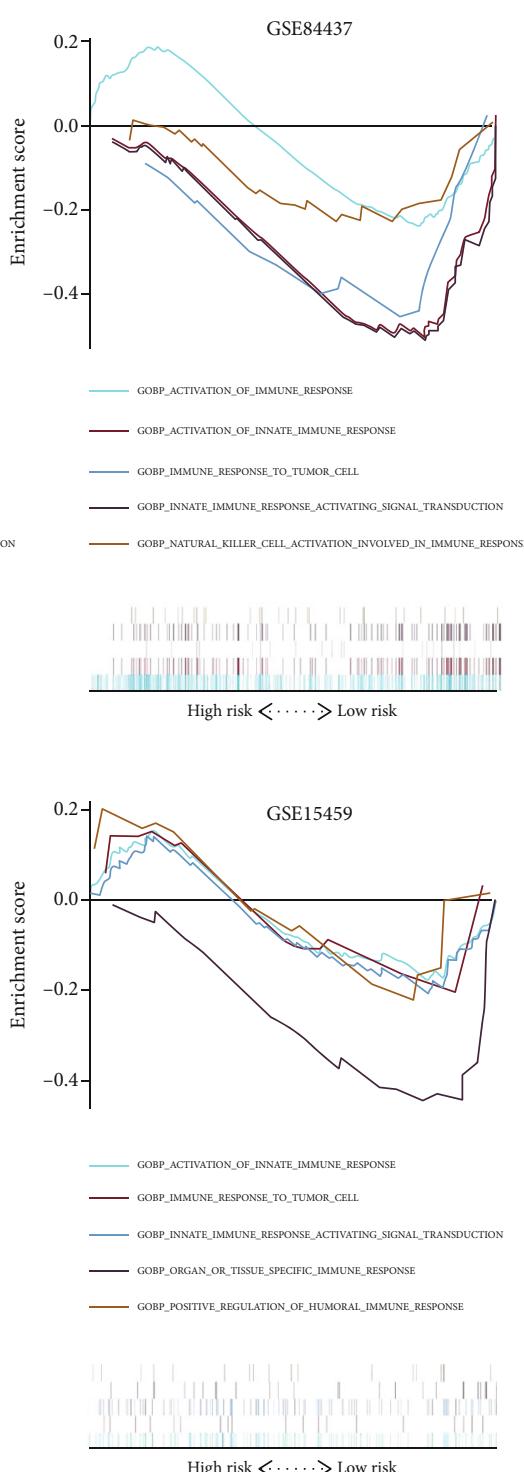

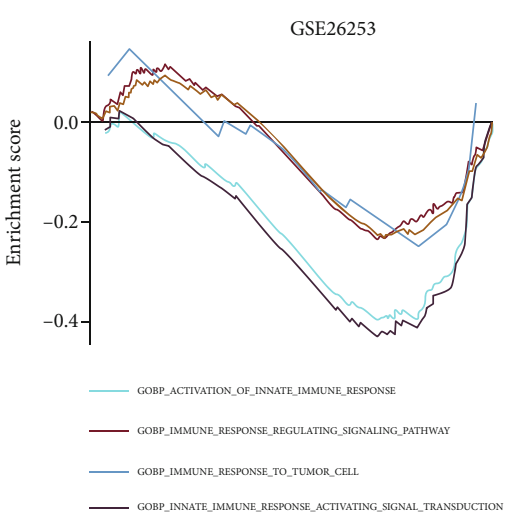

_ GOBP_POSTTIVE_REGULATION_OF_IMMUNE_RESPONSE

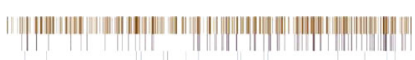

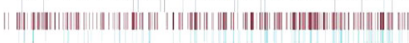
High risk $<\cdots\rangle$ Low risk
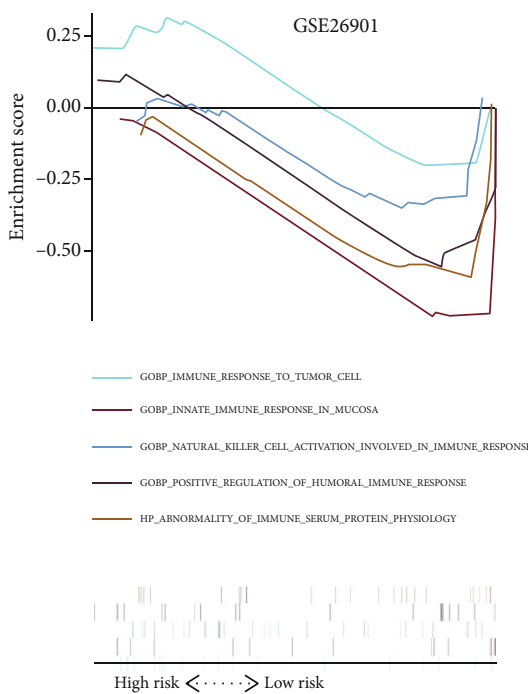

FIGURE 8: Gene set enrichment analysis for immune-related pathways in different risk groups.

found that the OS of 24 high-risk patients was significantly $(p<0.001)$ lower than that of 45 low-risk patients, and the time-dependent ROC analysis also demonstrated that the risk score had high accuracy (supplement material 3). Therefore, this evidence suggests that our model is a promising prognostic predictor for Asian patients with GC.

Next, we assessed the potential mechanism of the risk score with a focus on immune cell infiltration (ICI) and clinicopathology. The high-risk group presented a significant increase in the infiltration level of M2 macrophages, and M2 macrophages were a risk factor for GC prognosis. It is generally believed that M2 macrophages play a leading role in cancer progression and metastasis [27]. In addition, we identified three kinds of immune cells, resting NK cells, activated memory CD4 T cells, and activated dendritic cells, which were favorable for the prognosis of GC. They all showed a higher infiltration degree in the low-risk group, which has rarely been reported before. In terms of clinical features, we focused on the correlation between the risk score and the Lauren classification and clinicopathological stage. Lauren classification is a method that combines cell morphology and histochemistry to classify GC cells [28]. This method divided GC into the intestinal type and the diffuse type. Previous studies have shown that intestinal-type GC has a higher degree of differentiation, is more common in the elderly, has a low degree of malignancy, and has a better prognosis [29], while diffuse-type GC is often undifferentiated, has a poor prognosis, and is difficult to treat [30]. We found that the risk score of intestinal-type GC was low, and the prognosis was good, which was consistent with previous reports. In clinical practice, the most popular prognostic evaluation system for GC is still TNM staging [31]. We found that the risk score increased with increasing stage, indicating that the risk score was positively correlated with disease stage but negatively correlated with prognosis. In addition, the GSEA results showed that the activation of the immune 

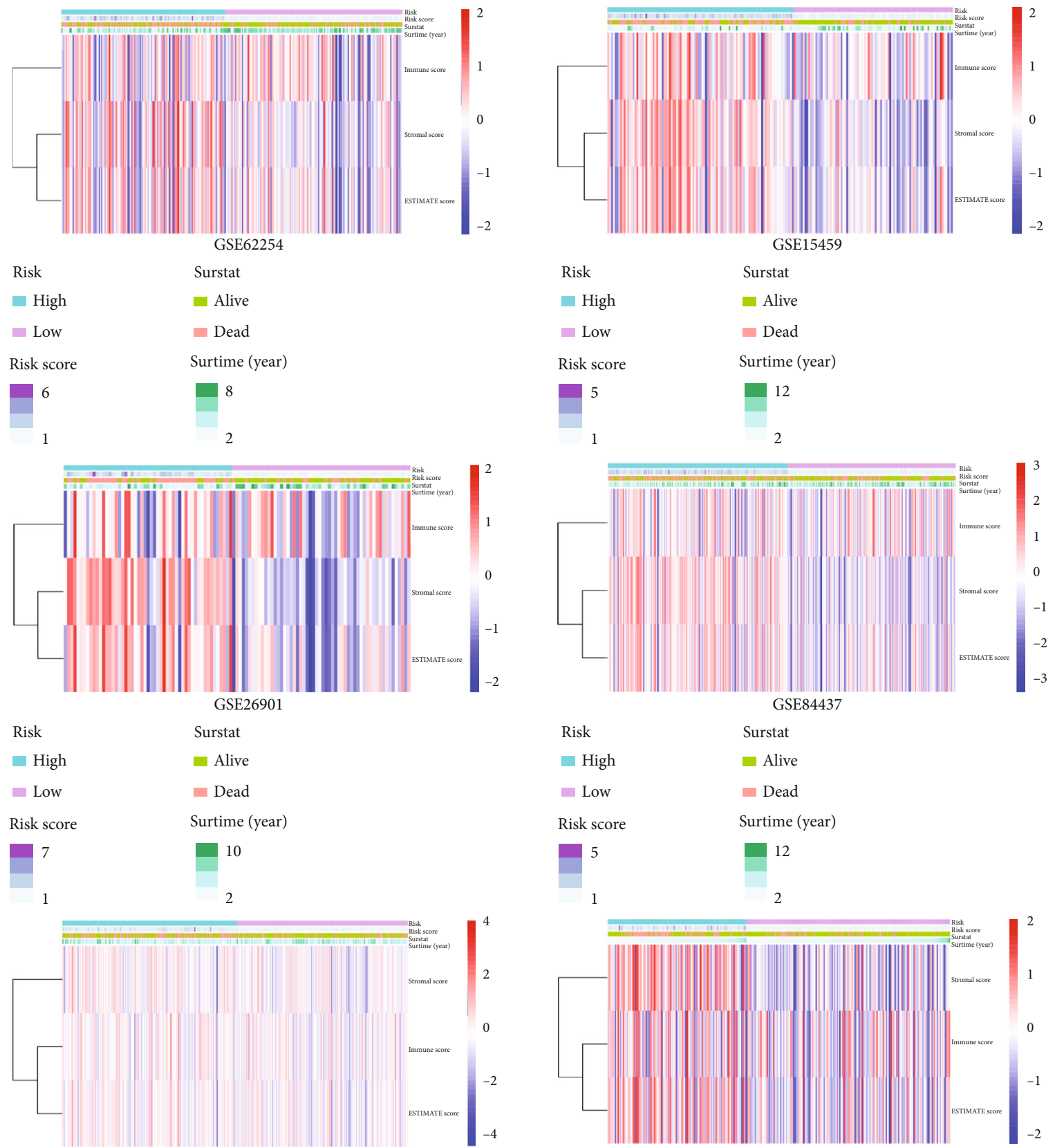
GSE26253
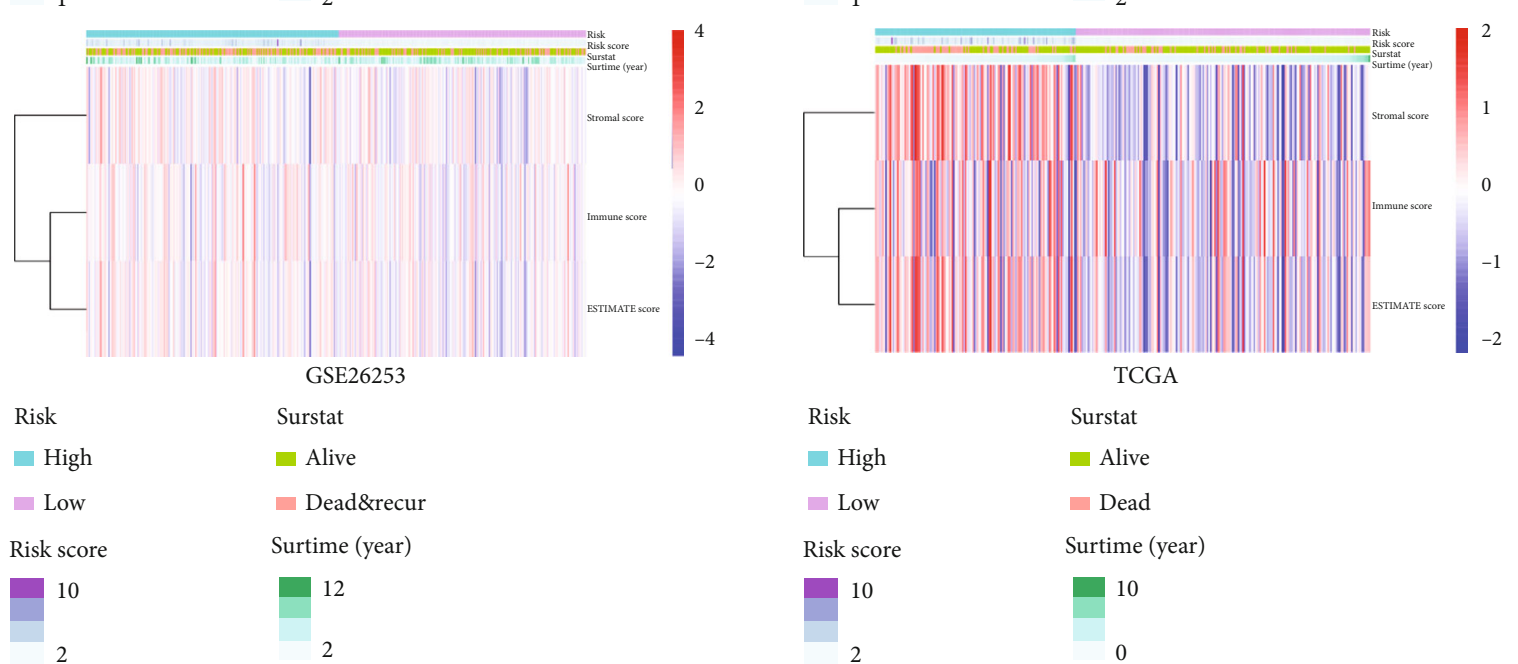

FIgURE 9: The presentation of ESTIMATE calculation results.

response in the high-risk group was weaker than that of the low-risk group, which indicated that the poor prognosis of the high-risk group may be related to immunosuppression. In terms of TME, the GC patients with higher Stromal Score had decreased OS and RFS, so targeted therapy of stromal cells in TME may have a positive effect on the prognosis of
GC patients. Considering the risk score exhibited a significantly positively correlation with the Stromal Score, we speculated that the higher risk score may facilitate the formation of TME to promote tumor proliferation and metastasis; the nine genes in the signature may be a promising target for the treatment of GC. 

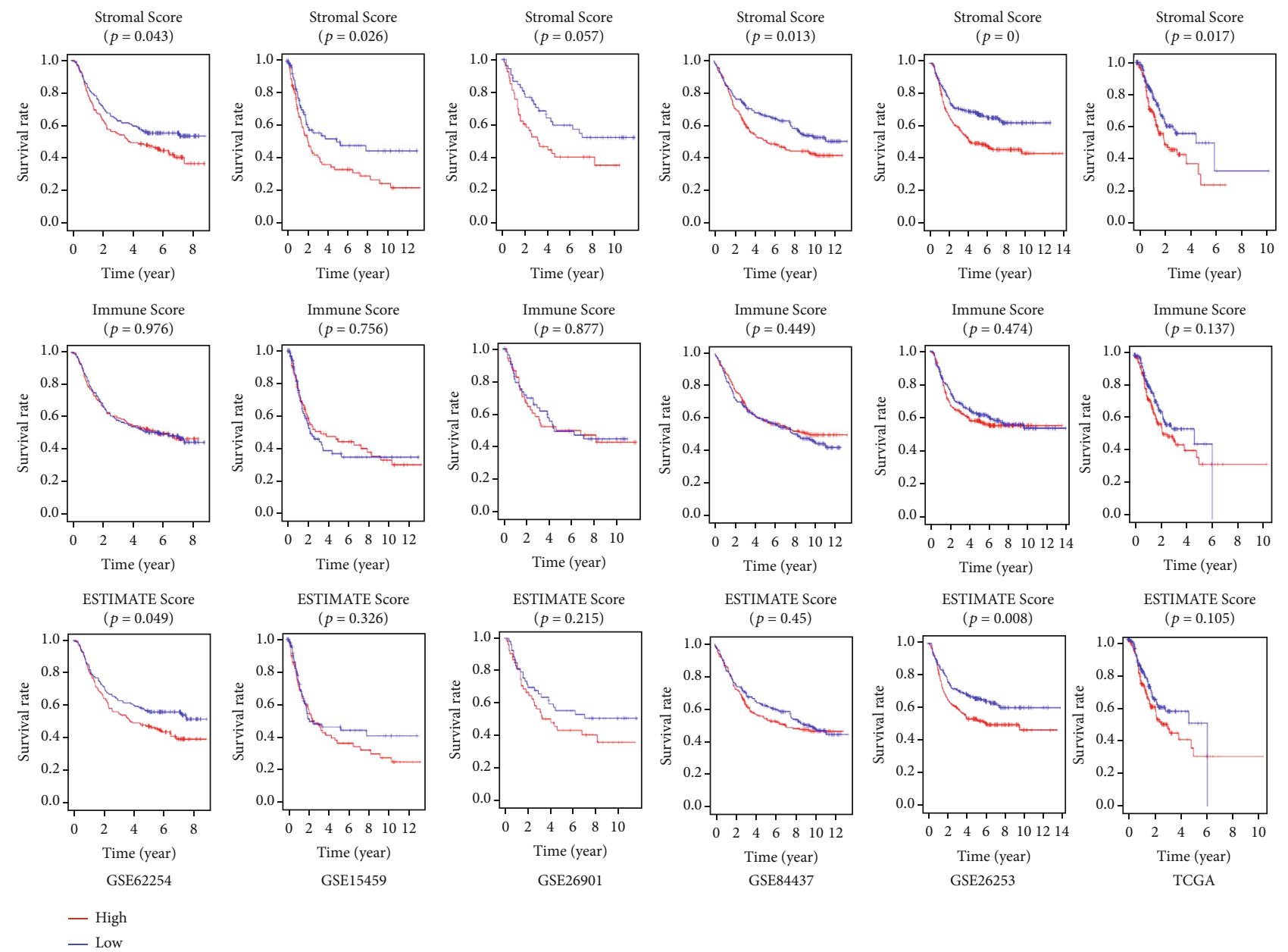

FIgURE 10: The Kaplan-Meier survival analysis of Stromal Score, Immune Score, and ESTIMATE Score in the six independent cohorts.

Therefore, compared with previous studies [32-34], this research has the following advantages. First, a total of 1799 GC patients from six independent datasets were included in this work, which was one of the largest GC prognostic model development projects to our knowledge. Second, the proposed signature has robustness in the prediction of the prognosis of GC through internal and external validation, especially for Asian GC patients. In view of the fact that the Asian region has the highest incidence and death of GC all over the world [35], the proposed prognostic model may receive increasing attention in the future. Third, we found the close correlation between the risk score and the stromal cells by investigating the TME, which was also a novel finding of this paper, considering similar reports were rare before, which may provide new clues for targeted therapy of stromal cells in GC.

Tumor cell-derived angiopoietin-like protein 2 (ANGPTL2) activates tumor cell motility, invasiveness, and epithelialmesenchymal transition to accelerate tumor metastasis in an autocrine/paracrine manner [36]. Recently, novel research indicated that stroma-derived ANGPTL2 could drive the production of immune-stimulated macrophages through the NF- $\kappa$ B pathway and accelerate the activation of CD4 T helper 1 (Th1) cells to play an antitumor role [37]. Increasing evi- dence has shown that connective tissue growth factor (CTGF) is a multifunctional signal regulator that promotes the occurrence, progression, and metastasis of cancer by regulating epithelial-mesenchymal transition (EMT), invasion, migration, cell proliferation, and drug resistance [38]. Esophageal cancer (ESCA) was accompanied by high upregulation of ESM1, which could be partly explained by cell proliferation and migration and the regulation of the Janus kinase (JAK) signaling pathway [39]. As a protein-coding gene, inhibin subunit $\beta \mathrm{B}$ (INHBB) is involved in the synthesis of transforming growth factor $-\beta$ (TGF- $\beta$ ) family members. Yuan et al. found that overexpression of INHBB largely contributed to macrophage infiltration and impeded the infiltration of memory $\mathrm{T}$ cells, mast cells, and dendritic cells in colorectal cancer and was correlated with worse OS and DFS [40]. According to Chen et al. [41], the growth and metastasis of an orthotopic hepatocellular carcinoma (HCC) tumor were promoted by the Sox9/INHBB axis, which activated peritumoral hepatic stellate cells (HSCs). NADPH oxidase 4 (NOX4) is a key regulator of reactive oxygen species production [42]. Ford et al. [43] reported that NOX4 is essential for maintaining the immunosuppressed tumor-associated fibroblast (CAF) phenotype in tumors. NOX4 inhibition could effectively overcome the resistance of CAF-mediated immunotherapy and 

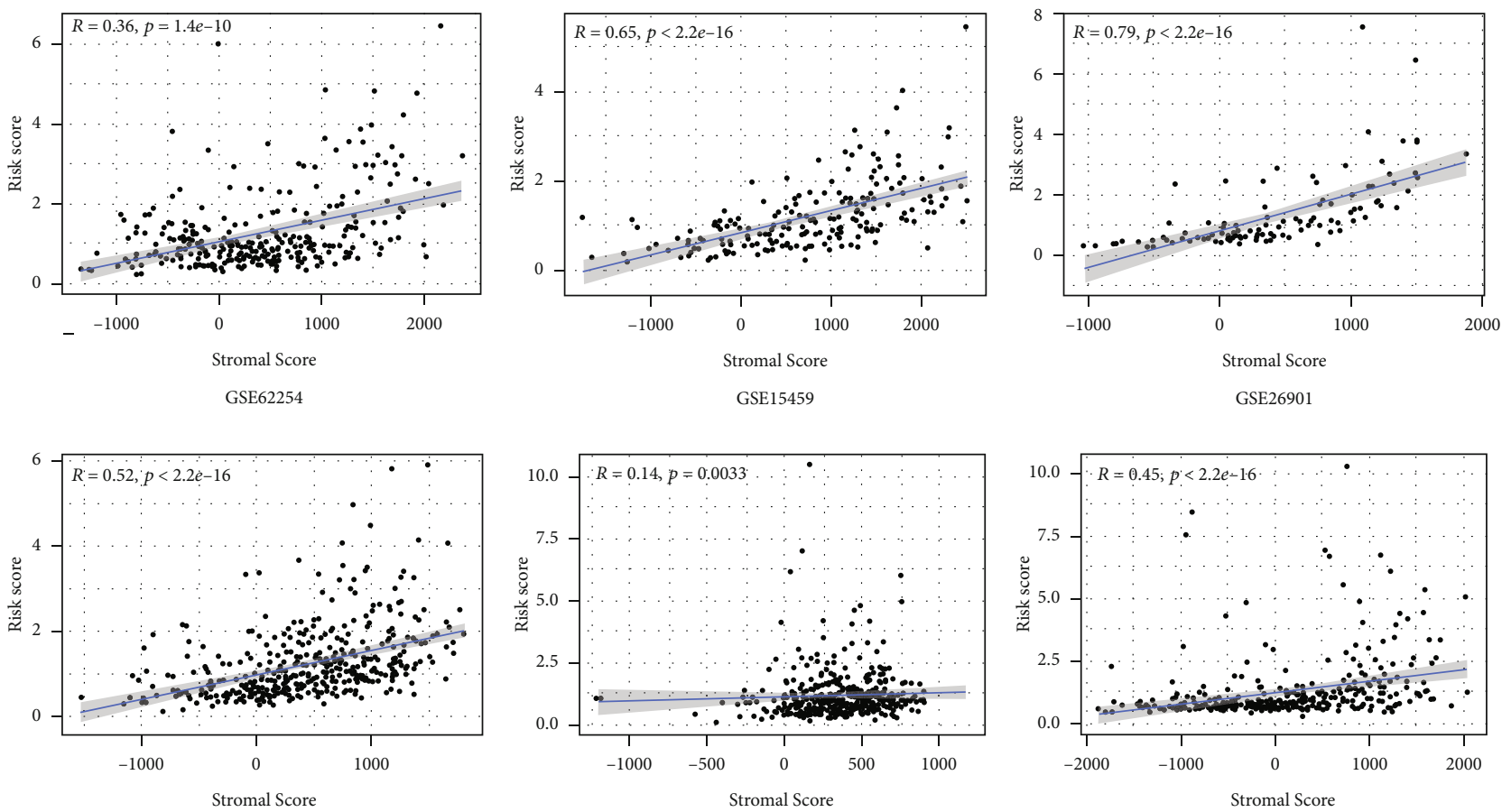

GSE84437
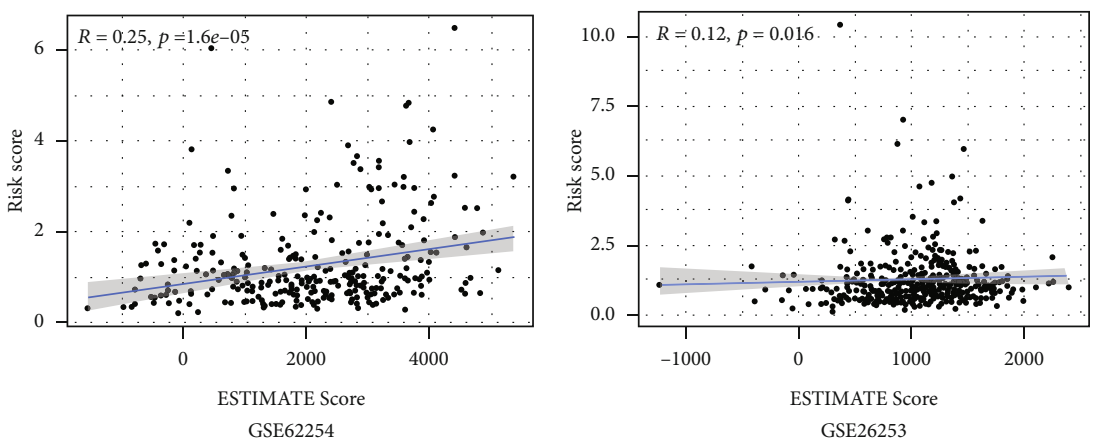

FIGURE 11: The association between risk score and the Stromal Score, Immune Score, and ESTIMATE Score.

improve the prognosis of many cancers. Sharanek et al. [44] found that the cytokine receptor for oncostatin M (OSMR) regulated the proliferation of glioblastoma by regulating oxidative phosphorylation to resist ionizing radiation. Retinol binding protein 1 (RBP1) plays a role in many physiological functions, such as regulating retinol transport and metabolism. Gao et al. [45] demonstrated that the overexpression of RBP1 promoted the growth, invasion, and migration of oral squamous cell carcinoma (OSCC) cells and that silencing RBP1 inhibited tumor formation in xenograft mice. SLIT2 glycoprotein has been described to regulate the inflammatory response and participate in autoimmune diseases [46]. The endothelial-derived SLIT2 protein, together with its receptor ROBO1, drove cancer cells to migrate and infiltrate into endothelial tissue. In mouse models of breast cancer and lung cancer, the deletion of SLIT2 inhibited metastasis. In contrast, when tumoral SLIT2 was blocked, metastatic progression was enhanced [47]. The results of Zhou et al.'s single-cell multiomics sequencing revealed that TPM2 was a fibroblast- specific biomarker associated with poor prognosis in colorectal cancer [48].

In this study, we systematically analyzed the prognostic value of immune-related genes in GC by integrating multiple sequencing datasets and clinical information. A risk score comprising 9 genes showed good performance in forecasting the prognosis of GC, and internal and external validation further confirmed its robustness. Since this study is retrospective, prospective clinical trials are still necessary in the future. The specific function of the nine genes in the progression of GC is still unknown and needs to be verified via further experiments.

\section{Conclusion}

In this study, we constructed and validated a robust prognostic signature for GC by integrating multiple sequencing datasets and clinical information, which may help improve the prognostic assessment system and treatment strategy for GC. 


\section{Abbreviations}

$\begin{array}{ll}\text { GC: } & \text { Gastric cancer } \\ \text { TCGA: } & \text { The Cancer Genome Atlas } \\ \text { GEO: } & \text { Gene Expression Omnibus } \\ \text { IRGs: } & \text { Immune-related genes } \\ \text { LASSO: } & \text { Least absolute shrinkage and selection } \\ & \text { operator } \\ \text { ROC: } & \text { Receiver operating characteristic } \\ \text { AUC: } & \text { Area under the curve } \\ \text { OS: } & \text { Overall survival } \\ \text { RFS: } & \text { Recurrence-free survival } \\ \text { ICI: } & \text { Immune cell infiltration } \\ \text { ESTIMATE: } & \text { Estimation of STromal and Immune cells in } \\ & \text { MAlignant Tumor tissues using Expression } \\ & \text { data } \\ \text { TME: } & \text { Tumor microenvironment } \\ \text { GSEA: } & \text { Gene set enrichment analysis } \\ \text { ES: } & \text { Enrichment score. }\end{array}$

\section{Data Availability}

The datasets analyzed for this study were obtained from the Gene Expression Omnibus (https://www.ncbi.nlm.nih.gov/ geo/), ImmPort database (https://immport.niaid.nih.gov), and The Cancer Genome Atlas (TCGA, https://portal.gdc .cancer.gov/).

\section{Conflicts of Interest}

The authors have no conflicts of interest to declare.

\section{Authors' Contributions}

Junyu Huo and Liqun Wu designed this study; Junyu Huo analyzed the data in this study and interpreted the findings and drafted the manuscript. Junyu Huo, Liqun $\mathrm{Wu}$, and Yunjin Zang carried out data management and revised the manuscript. All authors reviewed the final version of the manuscript. The manuscript is approved by all authors for publication.

\section{Supplementary Materials}

Supplement material 1: the detailed clinical information of meta-GEO cohorts. Supplement material 2: the comparison of the proposed signature with others' related reports. Supplement material 3: the time-dependent ROC curves. Supplement material 4: AJE language editing certificate. (Supplementary Materials)

\section{References}

[1] K. D. Crew and A. I. Neugut, "Epidemiology of gastric cancer," World journal of gastroenterology: WJG, vol. 12, no. 3, pp. 354362, 2006.

[2] E. Van Cutsem, X. Sagaert, B. Topal, K. Haustermans, and H. Prenen, "Gastric cancer," The Lancet, vol. 388, no. 10060, pp. 2654-2664, 2016.
[3] H. H. Hartgrink, E. P. Jansen, N. C. van Grieken, and C. J. van de Velde, "Gastric cancer," The Lancet, vol. 374, no. 9688, pp. 477-490, 2009.

[4] A. P. Thrift and H. B. El-Serag, "Burden of gastric cancer," Clinical Gastroenterology and Hepatology, vol. 18, no. 3, pp. 534-542, 2020.

[5] L. H. Eusebi, A. Telese, G. Marasco, F. Bazzoli, and R. M. Zagari, "Gastric cancer prevention strategies: a global perspective," Journal of gastroenterology and hepatology, vol. 35, no. 9, pp. 1495-1502, 2020.

[6] R. E. Sexton, M. N. Al Hallak, M. Diab, and A. S. Azmi, "Gastric cancer: a comprehensive review of current and future treatment strategies," Cancer and Metastasis Reviews, pp. 1-25, 2020.

[7] J. Machlowska, P. Kapusta, J. Baj et al., "High-throughput sequencing of gastric cancer patients: unravelling genetic predispositions towards an early-onset subtype," Cancers, vol. 12, no. 7, p. 1981, 2020.

[8] H. R. Kazmi, S. Kumari, S. Tiwari, A. Khanna, and G. Narayan, "Epigenetic mechanisms and events in gastric canceremerging novel biomarkers," Pathology \& Oncology Research, vol. 24, no. 4, pp. 757-770, 2018.

[9] T. N. Patel, S. Roy, and R. J. E. Ravi, "Gastric cancer and related epigenetic alterations,” vol. 11, 2017.

[10] W. Yang, J. Ma, W. Zhou et al., "Molecular mechanisms and theranostic potential of miRNAs in drug resistance of gastric cancer," Expert opinion on therapeutic targets, vol. 21, no. 11, pp. 1063-1075, 2017.

[11] J. Wang, S. Yu, G. Chen et al., "A novel prognostic signature of immune-related genes for patients with colorectal cancer," Journal of cellular and molecular medicine, vol. 24, pp. 84918504, 2020.

[12] B. Li, Y. Cui, M. Diehn, and R. Li, "Development and validation of an individualized immune prognostic signature in early-stage nonsquamous non-small cell lung cancer," JAMA oncology, vol. 3, pp. 1529-1537, 2017.

[13] J. Huo, L. Wu, and Y. Zang, "Development and validation of a novel immune-gene pairs prognostic model associated with CTNNB1 alteration in hepatocellular carcinoma," Medical Science Monitor, vol. 26, 2020.

[14] J. Huo, L. Wu, and Y. Zang, “A prognostic model of 15 immune-related gene pairs associated with tumor mutation burden for hepatocellular carcinoma," Frontiers in Molecular Biosciences, vol. 7, 2020.

[15] J. Huo, L. Wu, Y. Zang et al., "Eight-gene metabolic signature related with tumor-associated macrophages predicting overall survival for hepatocellular carcinoma," BMC cancer, vol. 21, pp. 1-15, 2021.

[16] J. Huo, L. Wu, and Y. Zang, "Development and validation of a CTNNB1-associated metabolic prognostic model for hepatocellular carcinoma," Journal of Cellular and Molecular Medicine, vol. 25, no. 2, pp. 1151-1165, 2021.

[17] J. Huo, L. Wu, and Y. Zang, "A robust nine-gene prognostic signature associated with tumour doubling time for hepatocellular carcinoma," Life Sciences, vol. 260, pp. 118396-118396, 2020.

[18] P. Charoentong, F. Finotello, M. Angelova et al., "Pan-cancer immunogenomic analyses reveal genotype-immunophenotype relationships and predictors of response to checkpoint blockade," Cell reports, vol. 18, pp. 248-262, 2017.

[19] A. Jemal, F. Bray, M. M. Center, J. Ferlay, E. Ward, and D. Forman, "Global cancer statistics," CA: a cancer journal for clinicians, vol. 61, no. 2, pp. 69-90, 2011. 
[20] A. D. Wagner, N. L. Syn, M. Moehler et al., "Chemotherapy for advanced gastric cancer," Cochrane database of systematic reviews, no. 8, 2017.

[21] T. Sano, D. G. Coit, H. H. Kim et al., "Proposal of a new stage grouping of gastric cancer for TNM classification: International Gastric Cancer Association staging project," Gastric cancer, vol. 20, pp. 217-225, 2017.

[22] T. L. Whiteside, "The tumor microenvironment and its role in promoting tumor growth," Oncogene, vol. 27, no. 45, pp. 5904-5912, 2008.

[23] R. Kim, "Cancer immunoediting: from immune surveillance to immune escape," in Cancer Immunotherapy, pp. 9-27, Elsevier, 2007.

[24] S. Yu, C. Hu, L. Cai et al., "Seven-gene signature based on glycolysis is closely related to the prognosis and tumor immune infiltration of patients with gastric cancer," Frontiers in oncology, vol. 10, 2020.

[25] R. Cristescu, J. Lee, M. Nebozhyn et al., "Molecular analysis of gastric cancer identifies subtypes associated with distinct clinical outcomes," Nature medicine, vol. 21, pp. 449-456, 2015.

[26] S. C. Oh, B. H. Sohn, J. H. Cheong et al., "Clinical and genomic landscape of gastric cancer with a mesenchymal phenotype," Nature communications, vol. 9, pp. 1-14, 2018.

[27] S. Aras and M. R. Zaidi, "TAMeless traitors: macrophages in cancer progression and metastasis," British journal of cancer, vol. 117, pp. 1583-1591, 2017.

[28] S. C. Ming, "Gastric carcinoma: a pathobiological classification,” Cancer, vol. 39, no. 6, pp. 2475-2485, 1977.

[29] M. Qiu, Y. Zhou, X. Zhang et al., "Lauren classification combined with HER2 status is a better prognostic factor in Chinese gastric cancer patients," BMC cancer, vol. 14, no. 1, pp. 1-8, 2014.

[30] J. Y. Lee, E. J. Gong, E. J. Chung et al., "The characteristics and prognosis of diffuse-type early gastric cancer diagnosed during health check-ups," Gut and liver, vol. 11, no. 6, p. 807, 2017.

[31] H. Jung, H. H. Lee, K. Y. Song, H. M. Jeon, and C. H. Park, "Validation of the seventh edition of the American Joint Committee on Cancer TNM staging system for gastric cancer," Cancer, vol. 117, pp. 2371-2378, 2011.

[32] S. Zhang, Z. Li, H. Dong et al., "Construction of an immunerelated gene signature to predict survival and treatment outcome in gastric cancer," Science Progress, vol. 104, no. 1, p. 0036850421997286, 2021.

[33] B. Jiang, Q. Sun, Y. Tong et al., "An immune-related gene signature predicts prognosis of gastric cancer," Medicine, vol. 98, no. $27,2019$.

[34] E. Zhao, C. Zhou, and S. Chen, "A signature of 14 immunerelated gene pairs predicts overall survival in gastric cancer," Clinical and Translational Oncology, pp. 1-10, 2020.

[35] A. Etemadi, S. Safiri, S. G. Sepanlou et al., "The global, regional, and national burden of stomach cancer in 195 countries, 1990-2017: a systematic analysis for the Global Burden of Disease study 2017," The Lancet Gastroenterology \& Hepatology, vol. 5, no. 1, pp. 42-54, 2020.

[36] H. Osumi, H. Horiguchi, T. Kadomatsu et al., "Tumor cellderived angiopoietin-like protein 2 establishes a preference for glycolytic metabolism in lung cancer cells," Cancer science, vol. 111, no. 4, p. 1241, 2020.

[37] H. Horiguchi, T. Kadomatsu, K. Miyata et al., "Stroma-derived ANGPTL2 establishes an anti-tumor microenvironment during intestinal tumorigenesis," Oncogene, pp. 1-13, 2020.
[38] Y. W. Shen, Y. D. Zhou, H. Z. Chen, X. Luan, and W. D. Zhang, "Targeting CTGF in cancer: an emerging therapeutic opportunity," Trends in Cancer, 2020.

[39] Y. Cui, W. Guo, Y. Li, J. Shi, S. Ma, and F. Guan, "Pan-cancer analysis identifies ESM1 as a novel oncogene for esophageal cancer," Esophagus, pp. 1-13, 2020.

[40] J. Yuan, A. Xie, Q. Cao, X. Li, and J. Chen, "INHBB is a novel prognostic biomarker associated with cancer-promoting pathways in colorectal cancer," BioMed research international, vol. 2020, 14 pages, 2020.

[41] Y. Chen, B. Qian, X. Sun et al., “Sox9/INHBB axis-mediated crosstalk between the hepatoma and hepatic stellate cells promotes the metastasis of hepatocellular carcinoma," Cancer Letters, vol. 499, pp. 243-254, 2020.

[42] A. Dasgupta, S. K. Shukla, E. Vernucci et al., "SIRT1-NOX4 signaling axis regulates cancer cachexia," Journal of Experimental Medicine, vol. 217, no. 7, 2020.

[43] K. Ford, C. J. Hanley, M. Mellone et al., "NOX4 inhibition potentiates immunotherapy by overcoming cancer-associated fibroblast-mediated CD8 T-cell exclusion from tumors," Cancer research, vol. 80, no. 9, pp. 1846-1860, 2020.

[44] A. Sharanek, A. Burban, M. Laaper et al., "OSMR controls glioma stem cell respiration and confers resistance of glioblastoma to ionizing radiation," Nature communications, vol. 11, pp. 1-16, 2020.

[45] L. Gao, Q. Wang, W. Ren et al., "The RBP1-CKAP4 axis activates oncogenic autophagy and promotes cancer progression in oral squamous cell carcinoma," Cell death \& disease, vol. 11 , no. 6 , pp. 1-16, 2020.

[46] Y. Zhang, L. Hu, X. Li, L. Chen, and X. Yang, "Slit2 is a potential biomarker for renal impairment in systemic lupus erythematosus," Clinical and Experimental Medicine, vol. 21, no. 1, pp. 63-71, 2021.

[47] B. Tavora, T. Mederer, K. J. Wessel et al., "Tumoural activation of TLR3-SLIT2 axis in endothelium drives metastasis," Nature, vol. 586, no. 7828, pp. 299-304, 2020.

[48] Y. Zhou, S. Bian, X. Zhou et al., "Single-cell multiomics sequencing reveals prevalent genomic alterations in tumor stromal cells of human colorectal cancer," Cancer Cell, vol. 38, no. 6, pp. 818-828. e5, 2020. 